\title{
Cine Substitution with Arylzinc Reagents: Scope and Mechanistic Studies
}

Santiago Barroso, ${ }^{\star \dagger}$ Sébastien Lemaire, ${ }^{\dagger}$ Vittorio Farina, ${ }^{\dagger}$ Andreas K. Steib, ${ }^{\S}$ Romain Blanc,${ }^{\S}$ and Paul Knochel $^{\S}$

${ }^{\dagger}$ Pharmaceutical Development and Manufacturing Sciences, Janssen Pharmaceutica, Turnhoutseweg 30, 2340 Beerse, Belgium

$\S$ Department of Chemistry and Biochemistry, Ludwig-Maximilians University of München, Butenandtstrasse 5-12, Haus F, 81377 München, Germany

\section{Contents}

GCMS spectra 1

\section{GCMS spectra}

\section{Experiments in Scheme 3}

MS spectra of selected chromatographic peaks (GC) are shown. Three types of quenches were used to distinguish the actual product distribution for the different pathways. Main mass peaks results from fragmentation of the pyranose ring and/or pivaloyl groups, being the aromatic side unaffected (labeling is preserved).

$\mathrm{NH}_{4} \mathrm{Cl}$ quench:

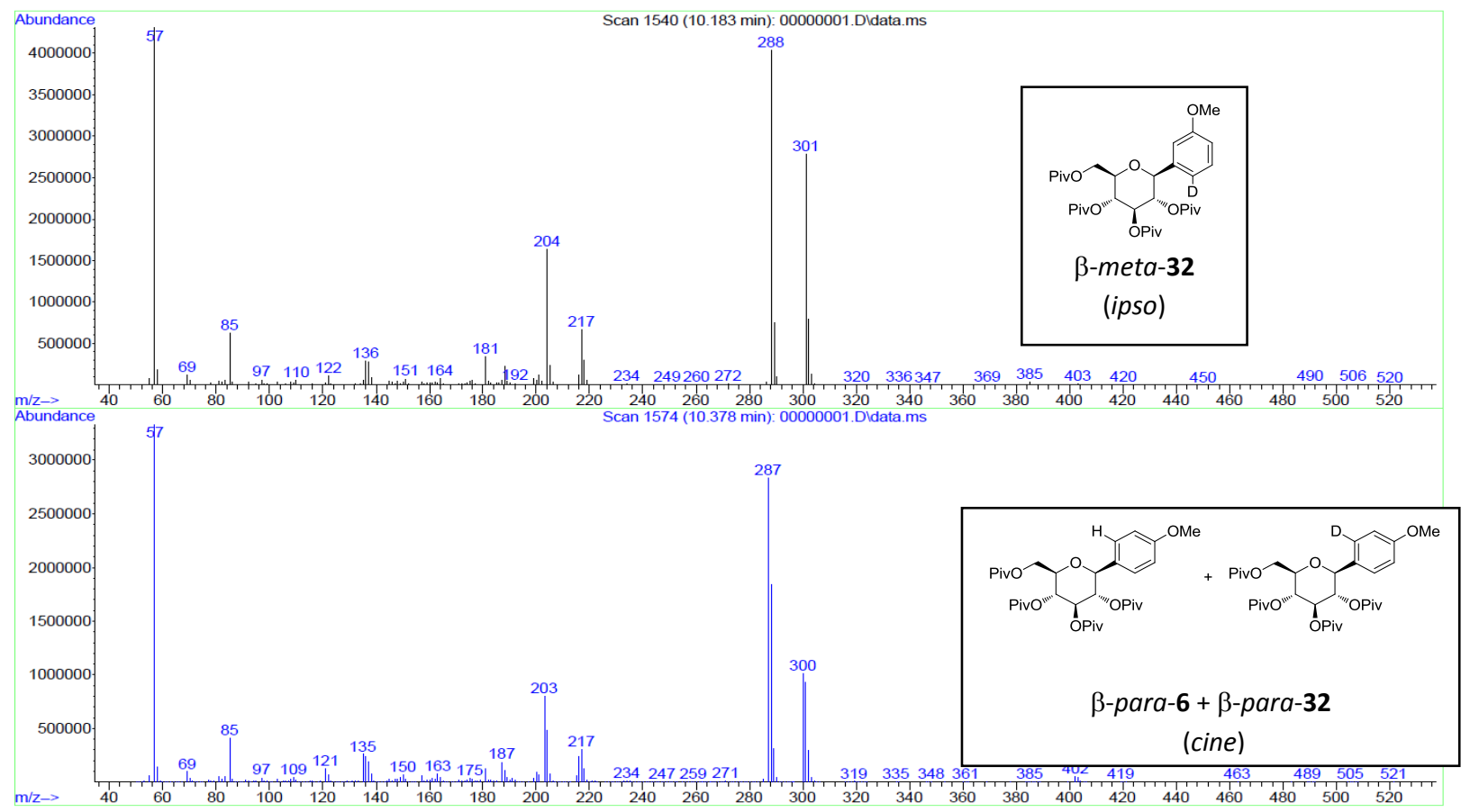




\section{MeOD quench}

Abundance

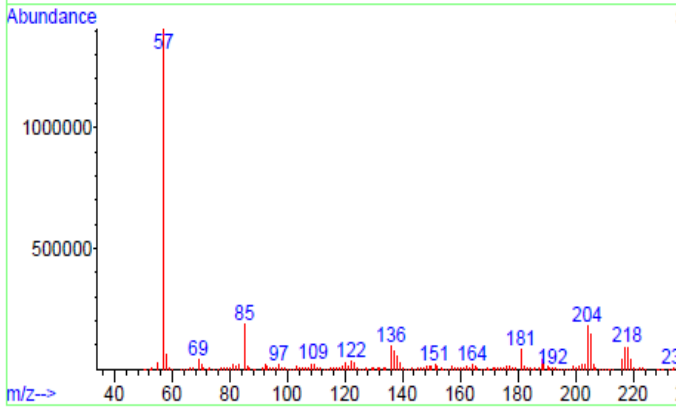

Scan 1494 (9.591 min): 00000001.DIda

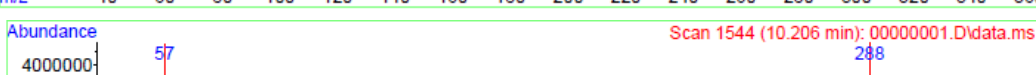

4000000

3000000

2000000

1000000

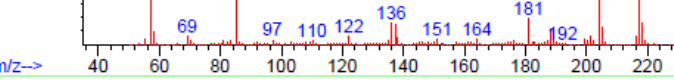

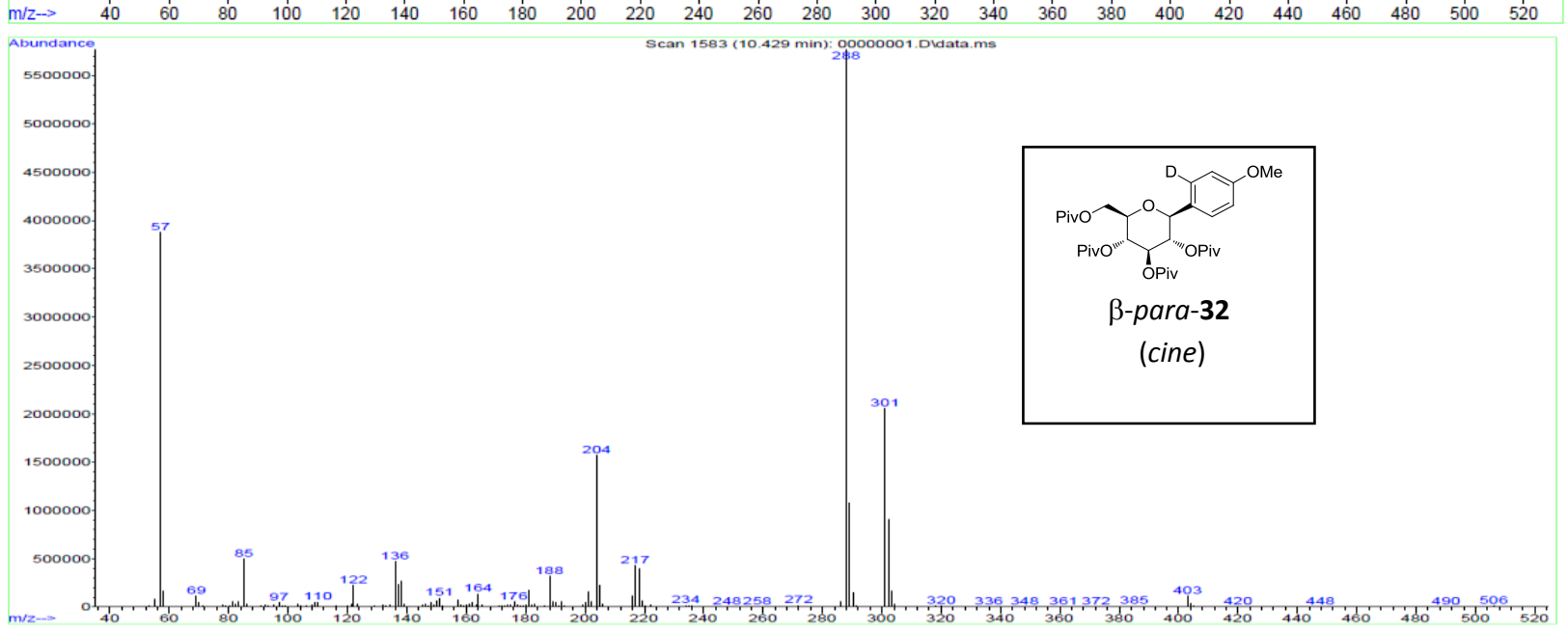

\section{$\mathrm{I}_{2}$ quench}

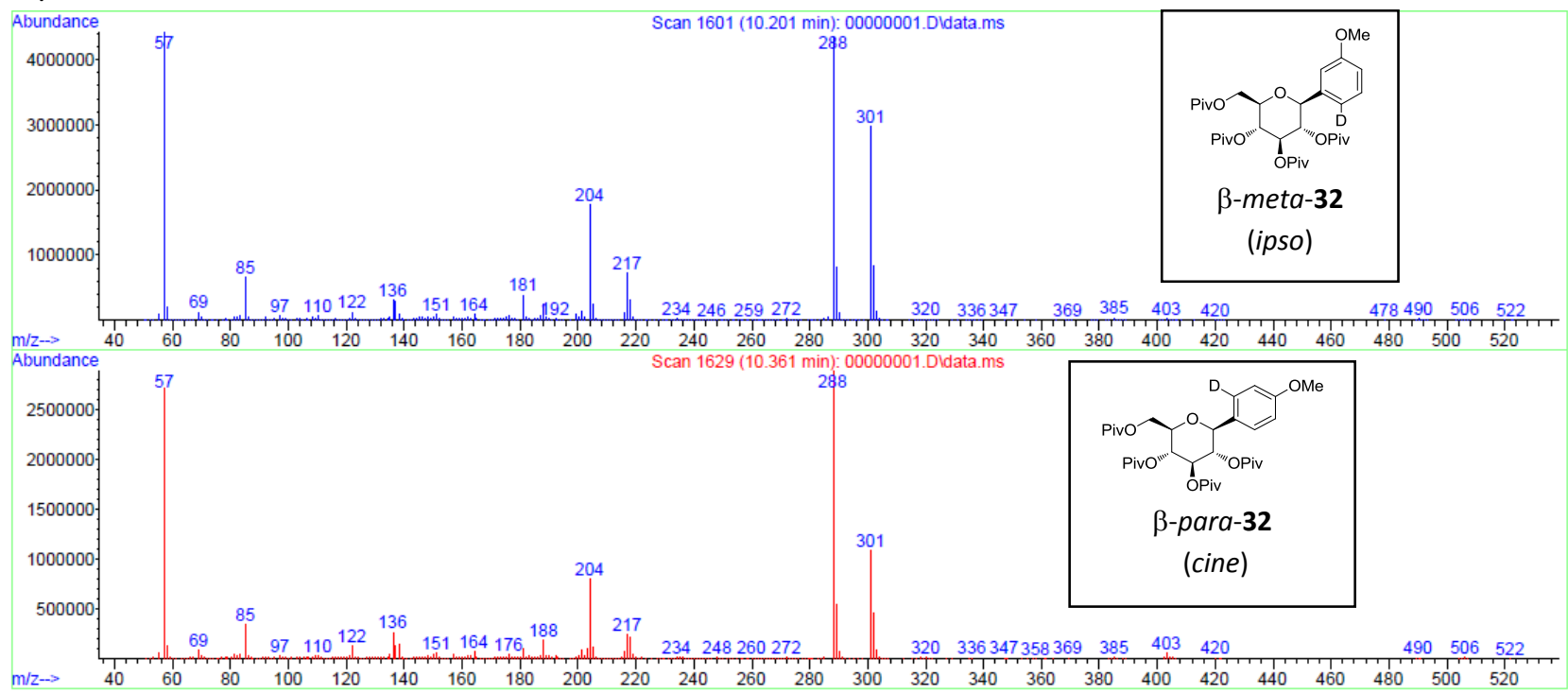




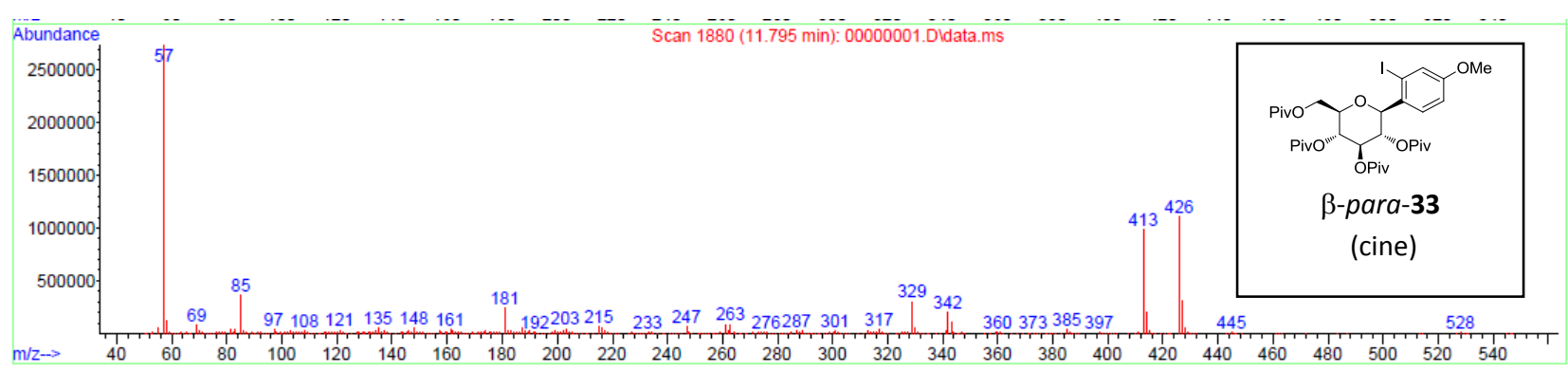

\section{Crossover experiment in Scheme 4}

Mass spectra (GCMS) corresponding to the mixture of the different meta and para products are shown below.

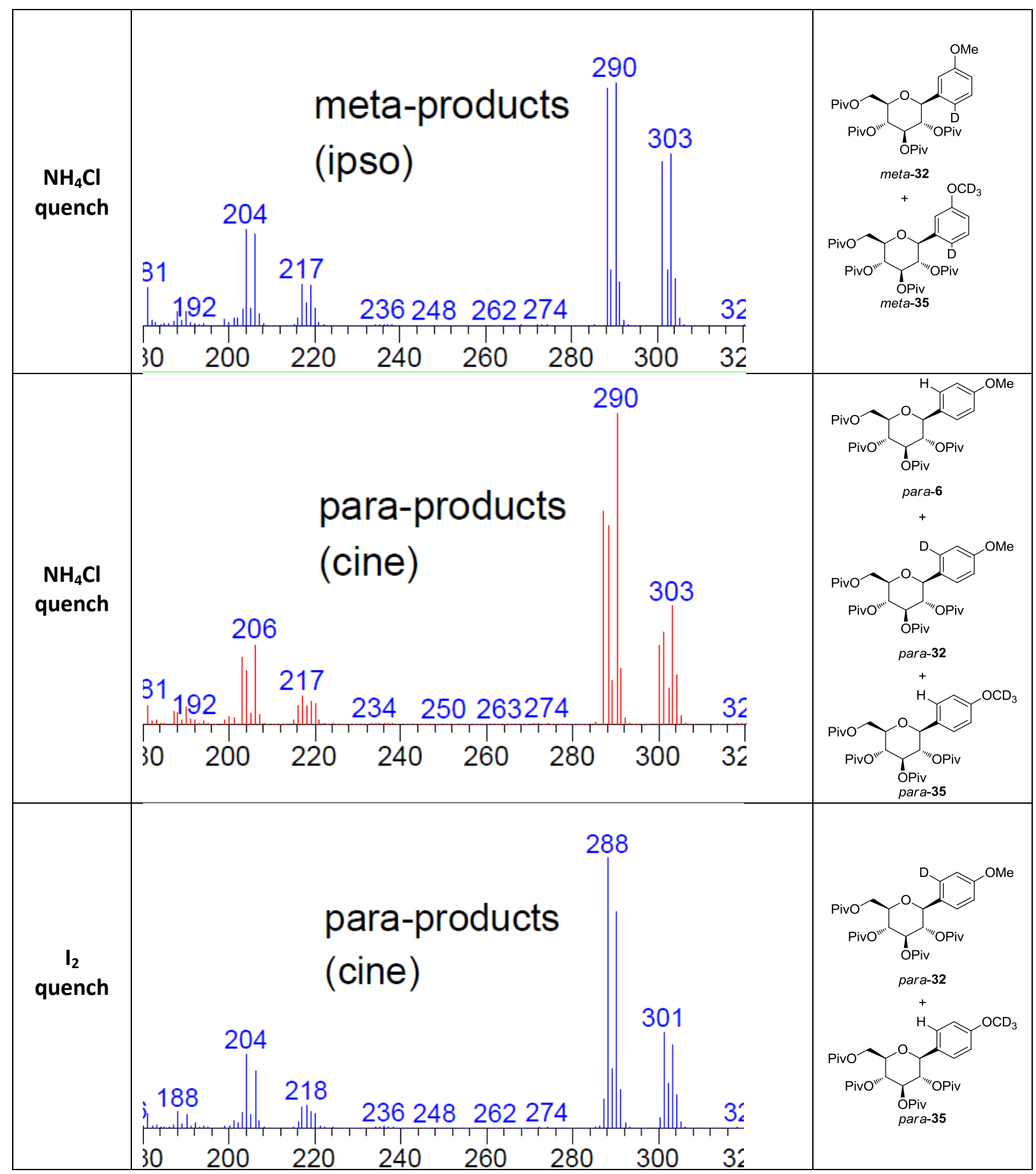




\section{Dilution experiments in Table 7}

Representative mass spectrum (LCMS) of a mixture of $\mathbf{3 8}$ and $\mathbf{3 9 .}$

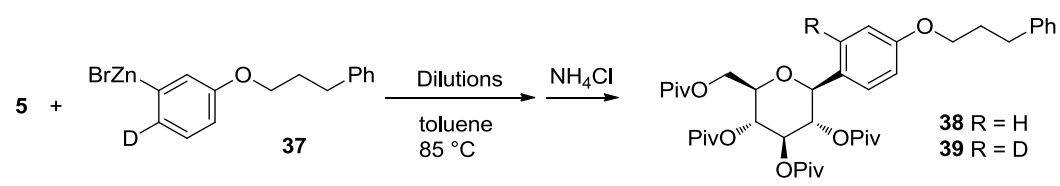

\section{SBARROSO-01-177-A}

Analyst: GWIN

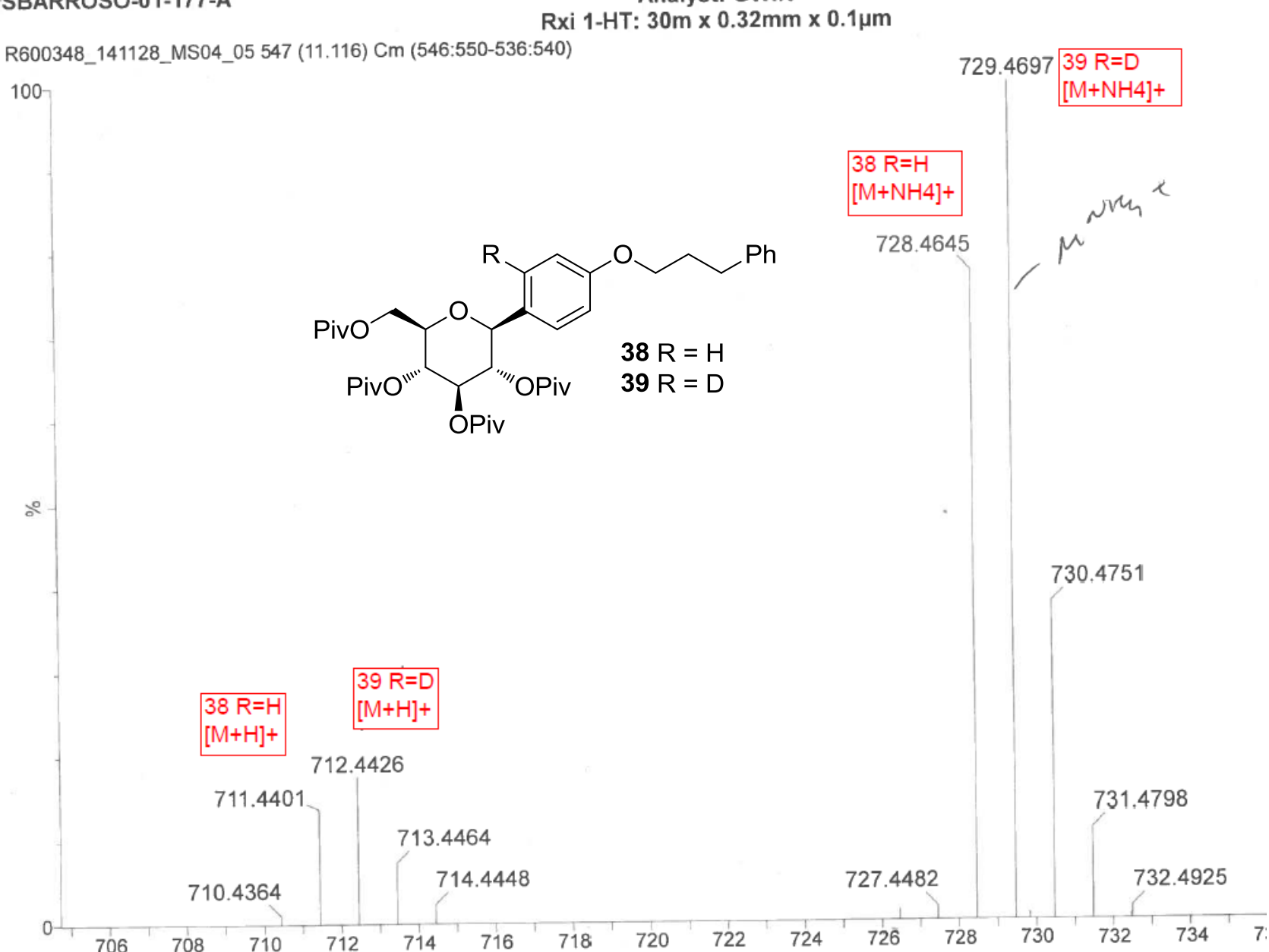




\section{NMR Spectra}

2,3,4,6-Tetra-O-pivaloyl 1-(4-anisyl)-1-deoxy- $\beta$-D-glucopyranose ( $\beta$-meta-6):

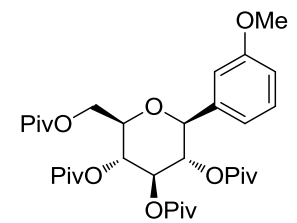

Chemical Formula: $\mathrm{C}_{33} \mathrm{H}_{50} \mathrm{O}_{10}$

Exact Mass: 606.3404

Molecular Weight: 606.7441
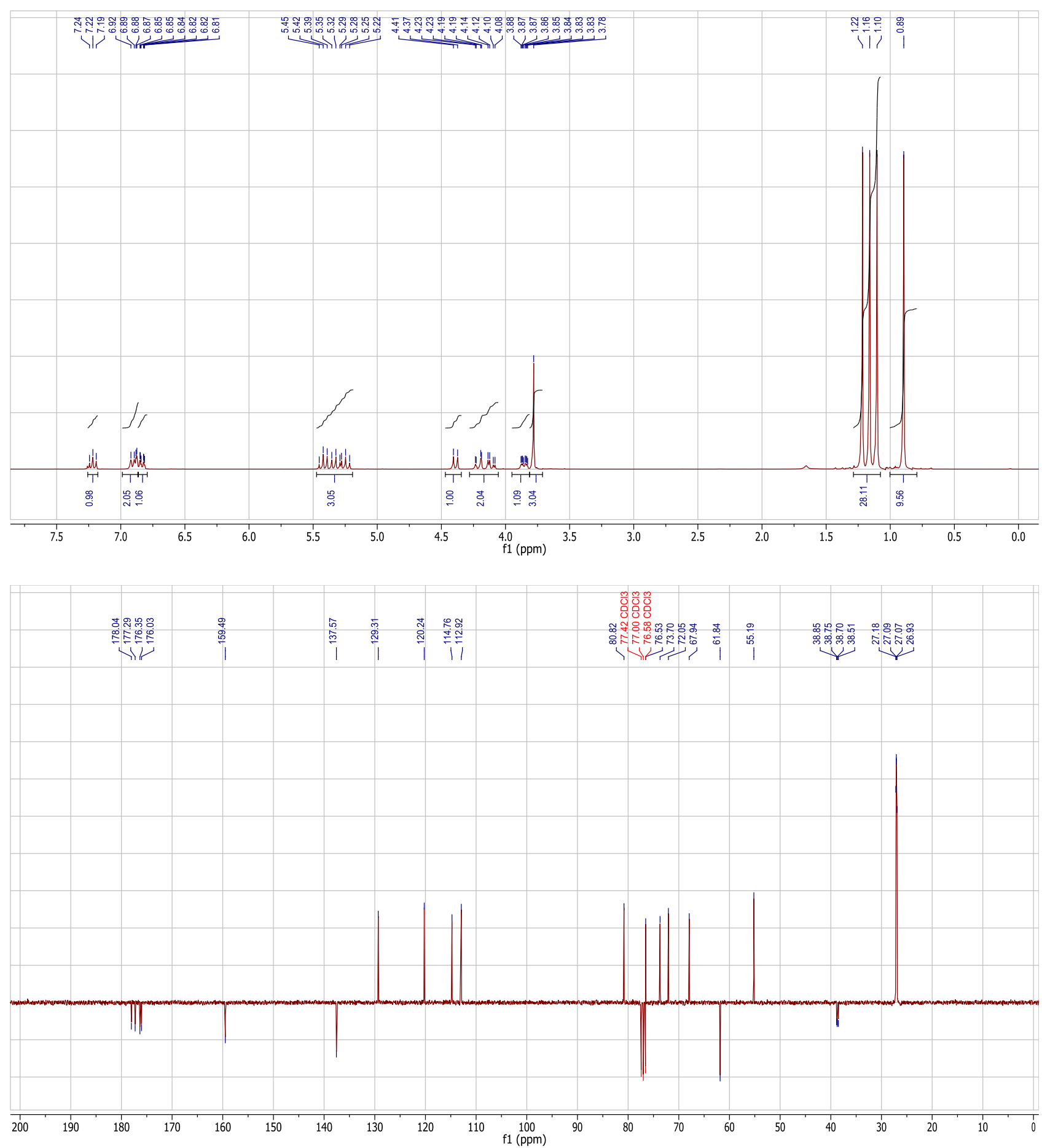
2,3,4,6-Tetra-O-pivaloyl 1-(2,4-dimethoxyphenyl)-1-deoxy- $\beta$-D-glucopyranose ( $\beta$-19):
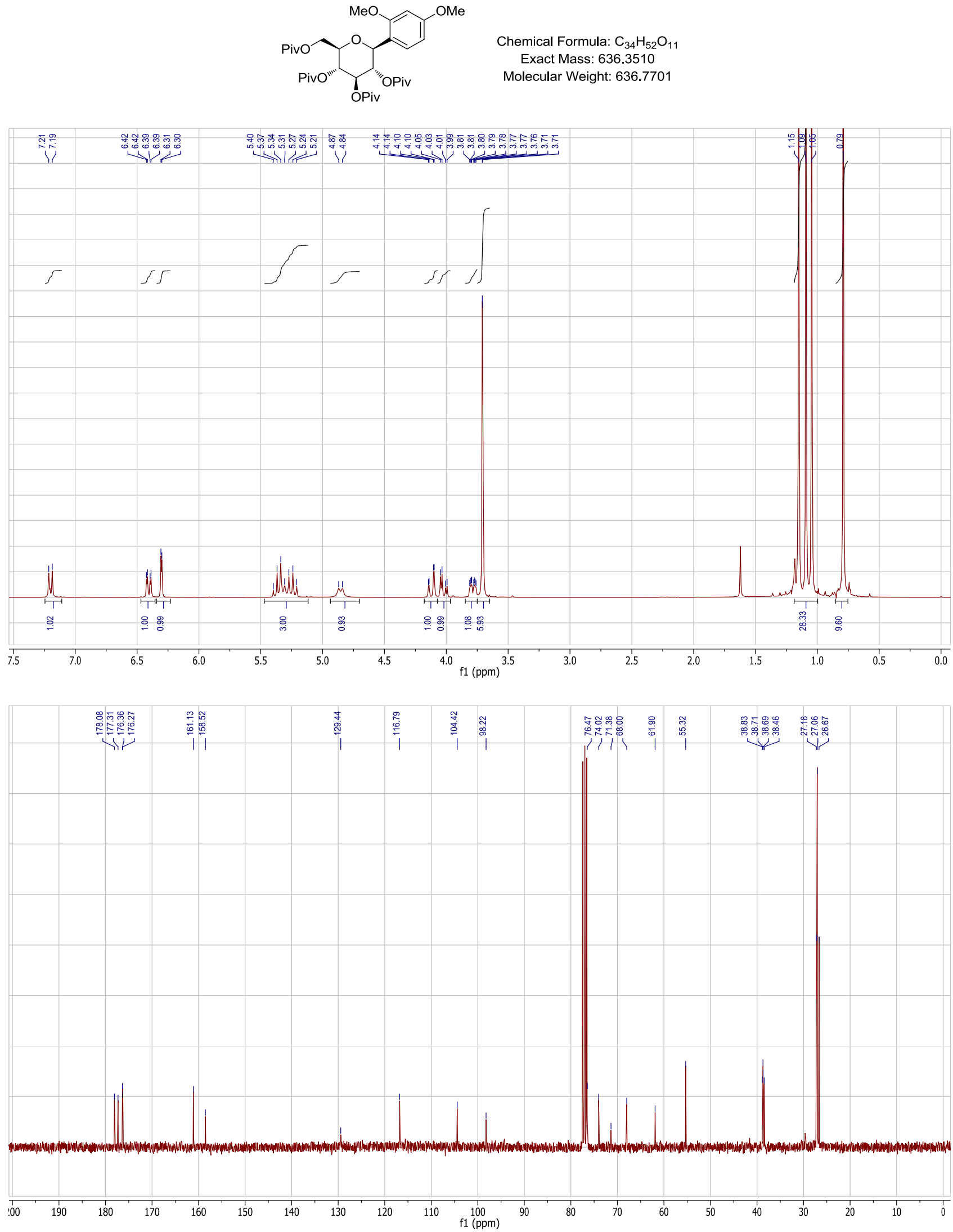
2,3,4,6-Tetra-O-pivaloyl 1-(biphenyl-3-yl)-1-deoxy- $\beta$-D-glucopyranose ( $\alpha$-meta-21):
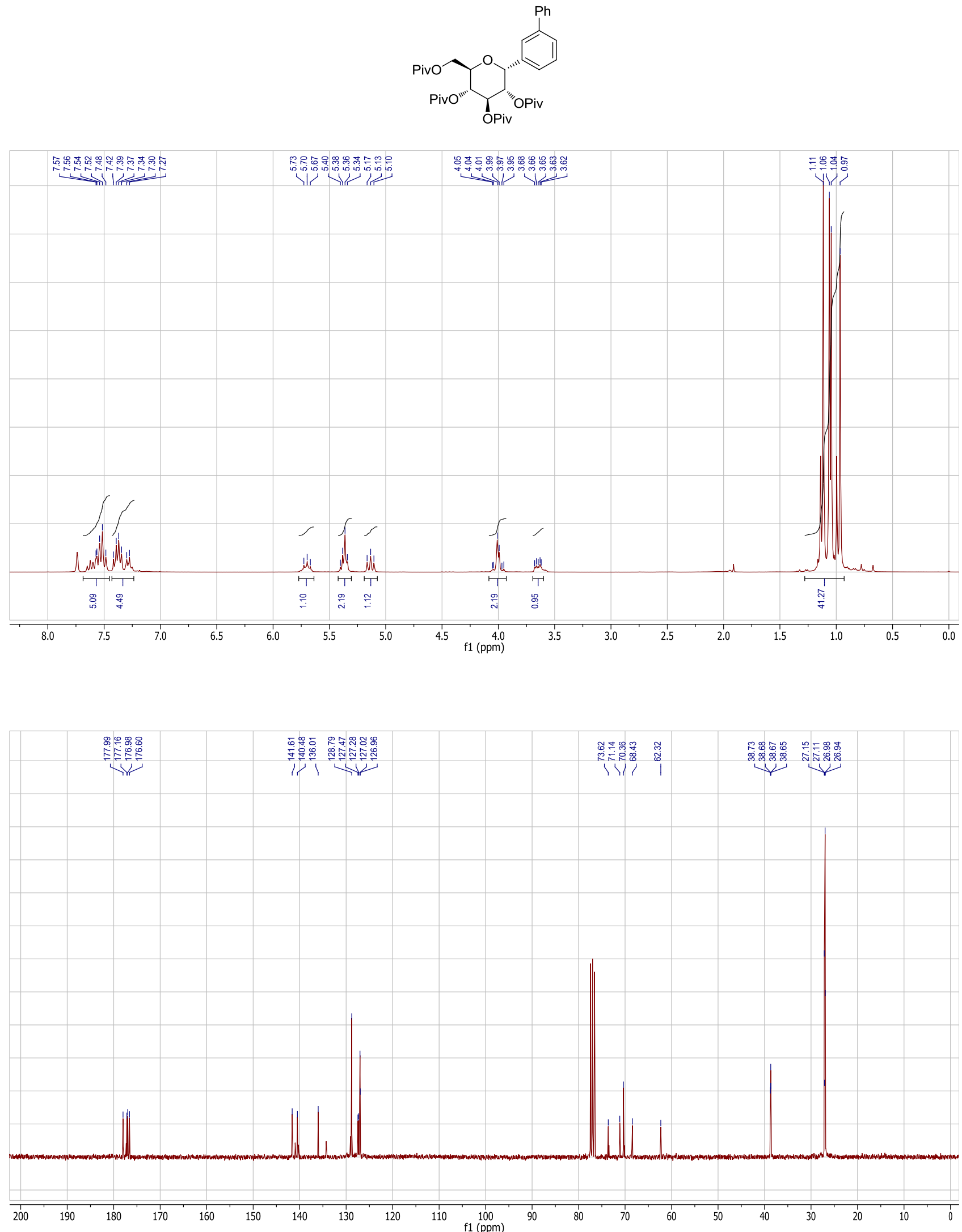
2,3,4,6-Tetra-O-pivaloyl 1-(biphenyl-3-yl)-1-deoxy- $\beta$-D-glucopyranose ( $\beta$-meta-21):

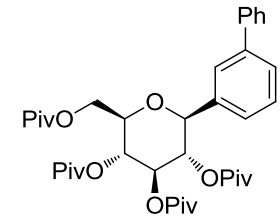

Chemical Formula: $\mathrm{C}_{38} \mathrm{H}_{52} \mathrm{O}_{9}$

Exact Mass: 652.3611

Molecular Weight: 652.8141
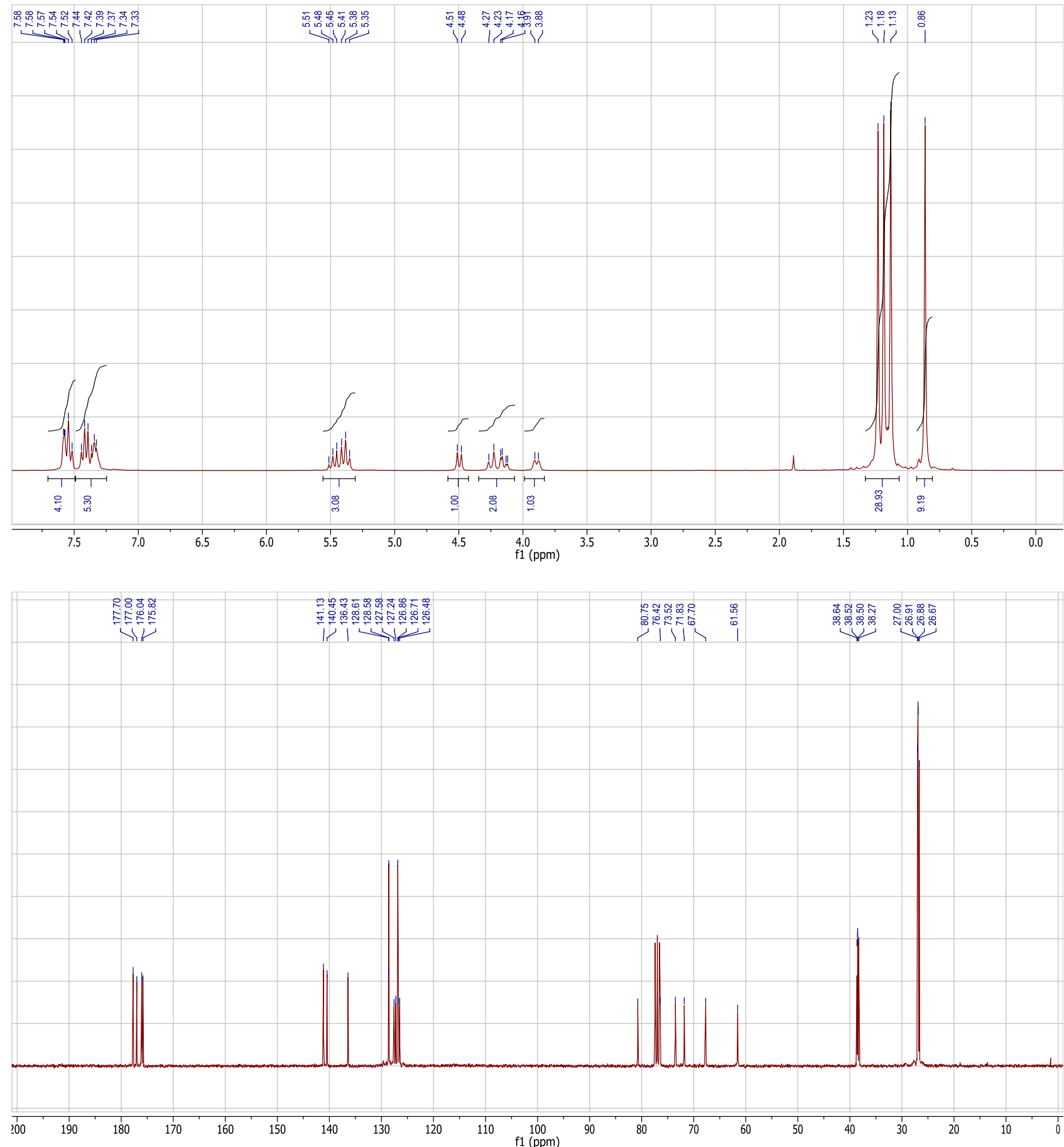
2,3,4,6-Tetra-O-pivaloyl 1-(biphenyl-4-yl)-1-deoxy- $\beta$-D-glucopyranose ( $\alpha$-para-21):
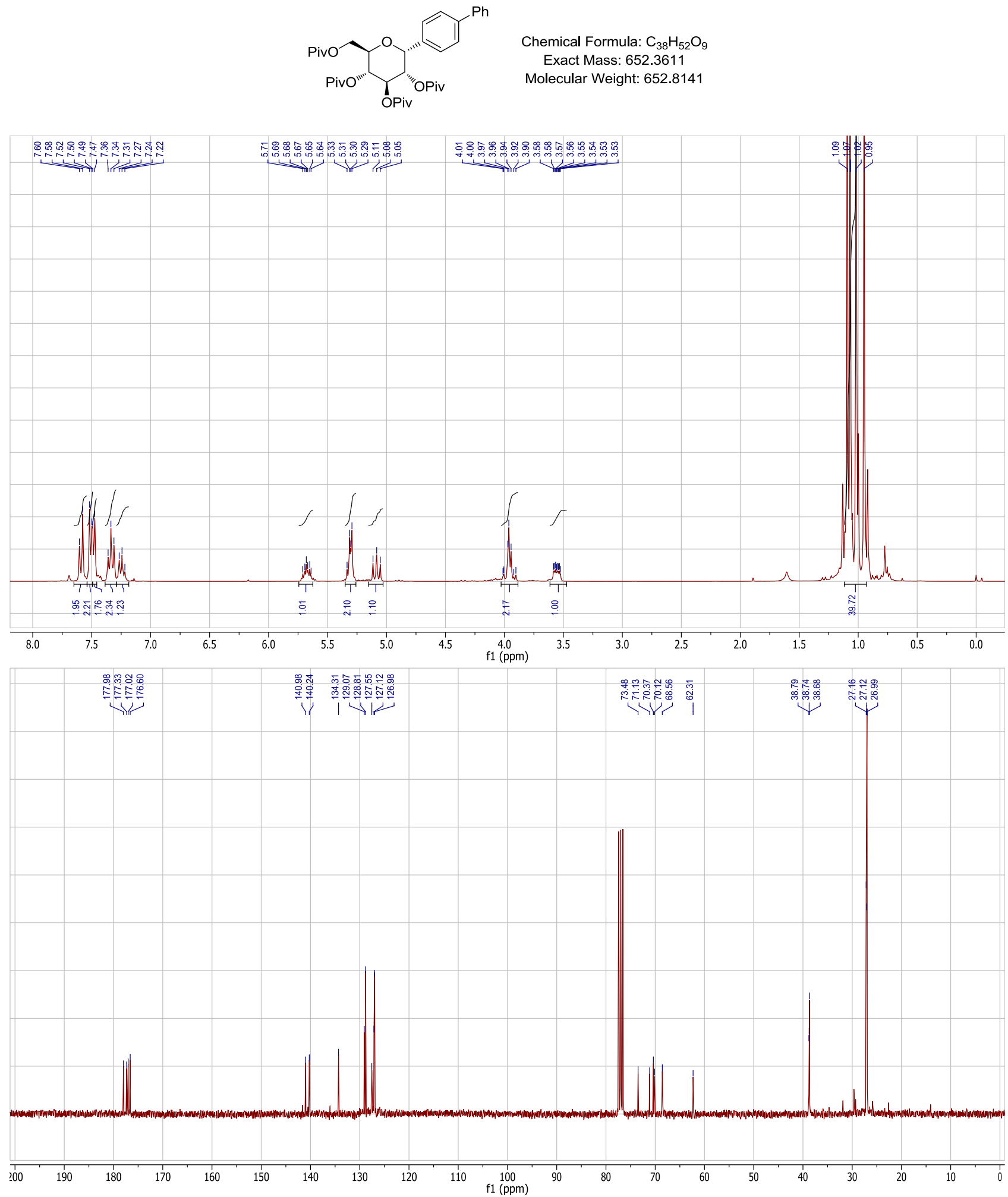
2,3,4,6-Tetra-O-pivaloyl 1-(biphenyl-4-yl)-1-deoxy- $\beta$-D-glucopyranose ( $\beta$-para-21):
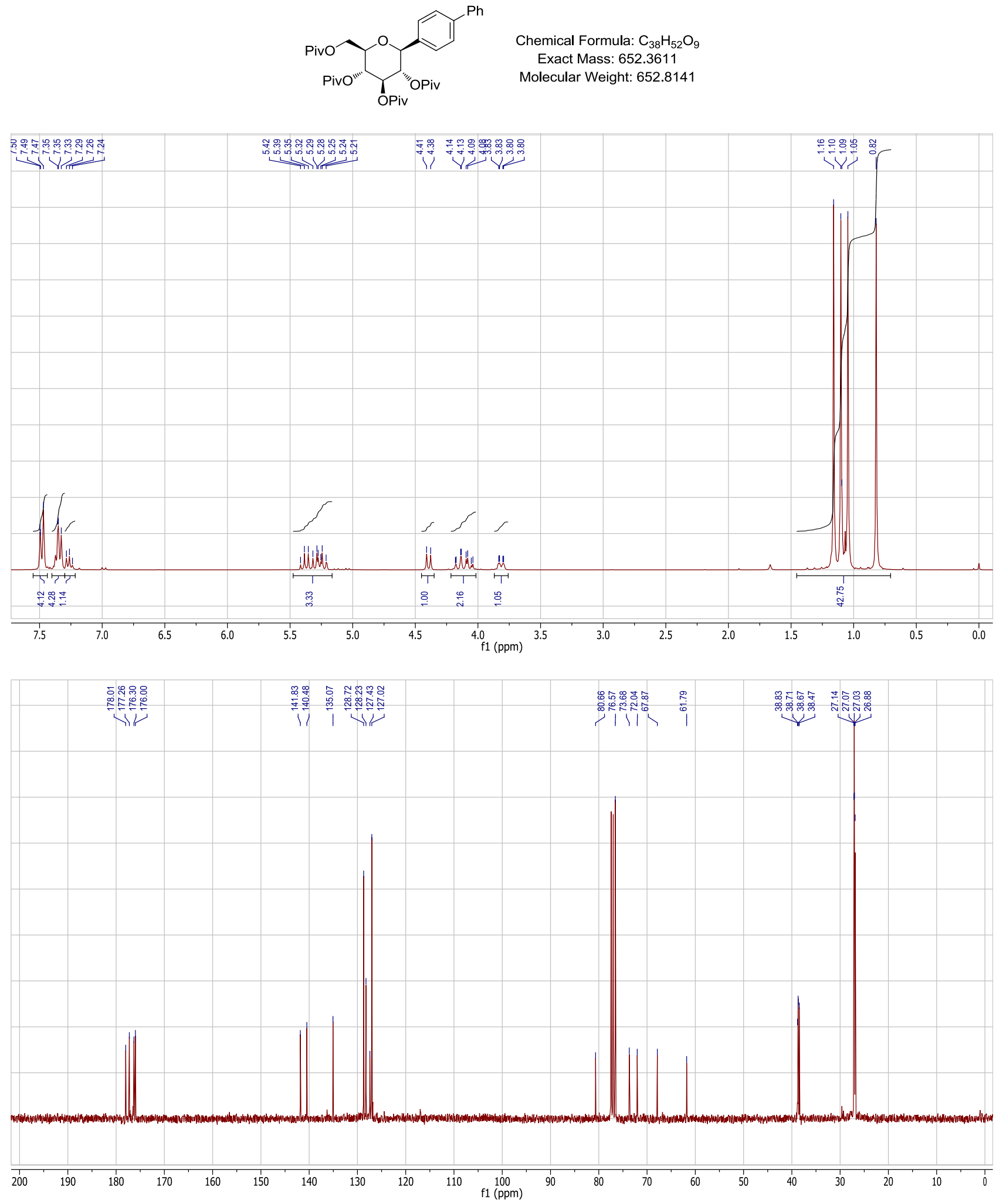
2,3,4,6-Tetra-O-pivaloyl 1-(3-toluyl)-1-deoxy- $\beta$-D-glucopyranose ( $\beta$-meta-22):

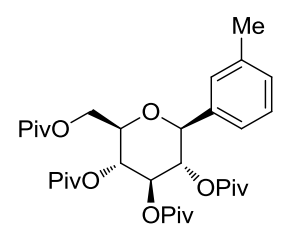

Chemical Formula: $\mathrm{C}_{33} \mathrm{H}_{50} \mathrm{O}_{9}$

Exact Mass: 590.3455

Molecular Weight: 590.7447
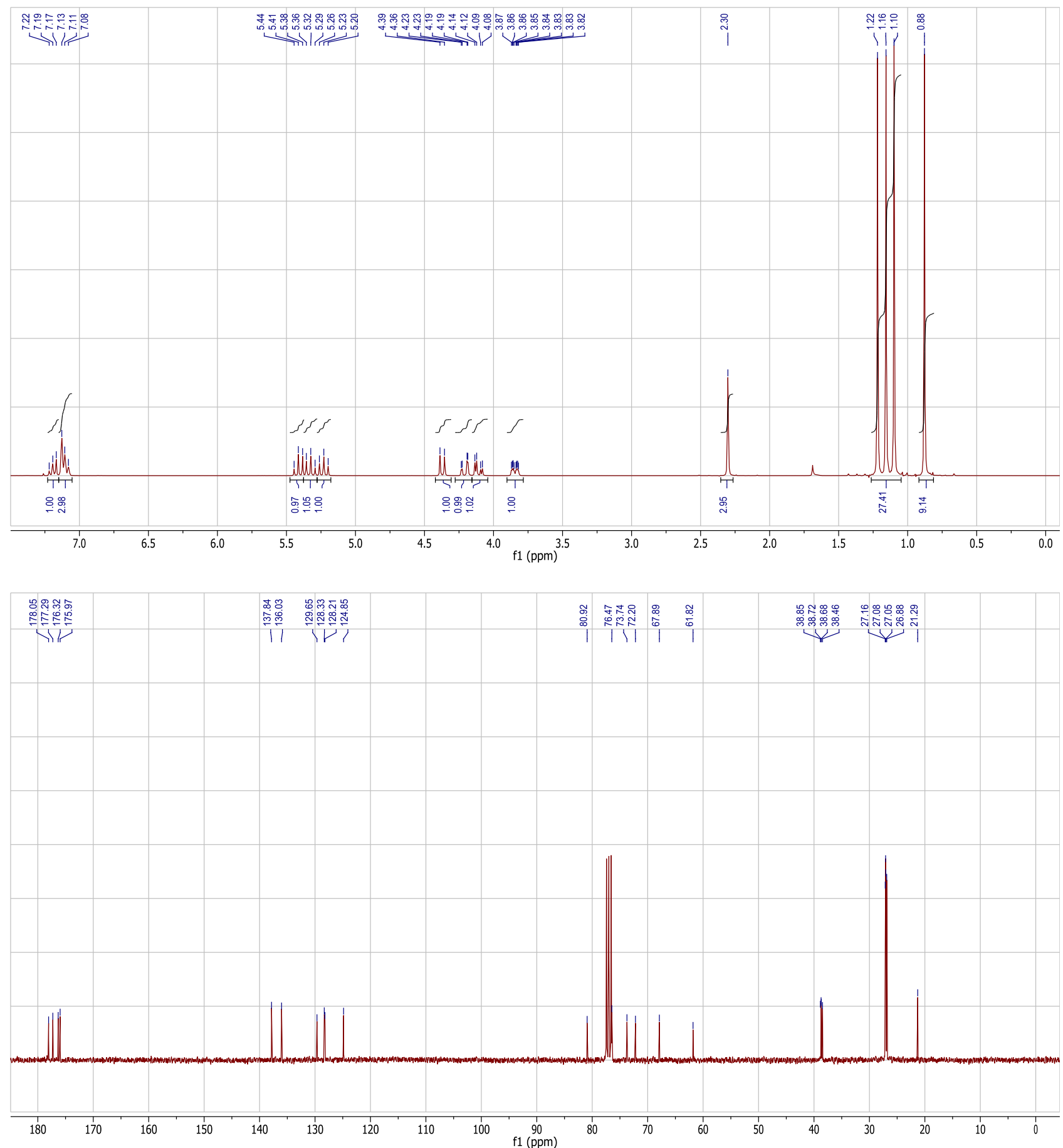
2,3,4,6-Tetra-O-pivaloyl 1-(2,4-dimethylphenyl)-1-deoxy- $\beta$-D-glucopyranose ( $\beta$-23):
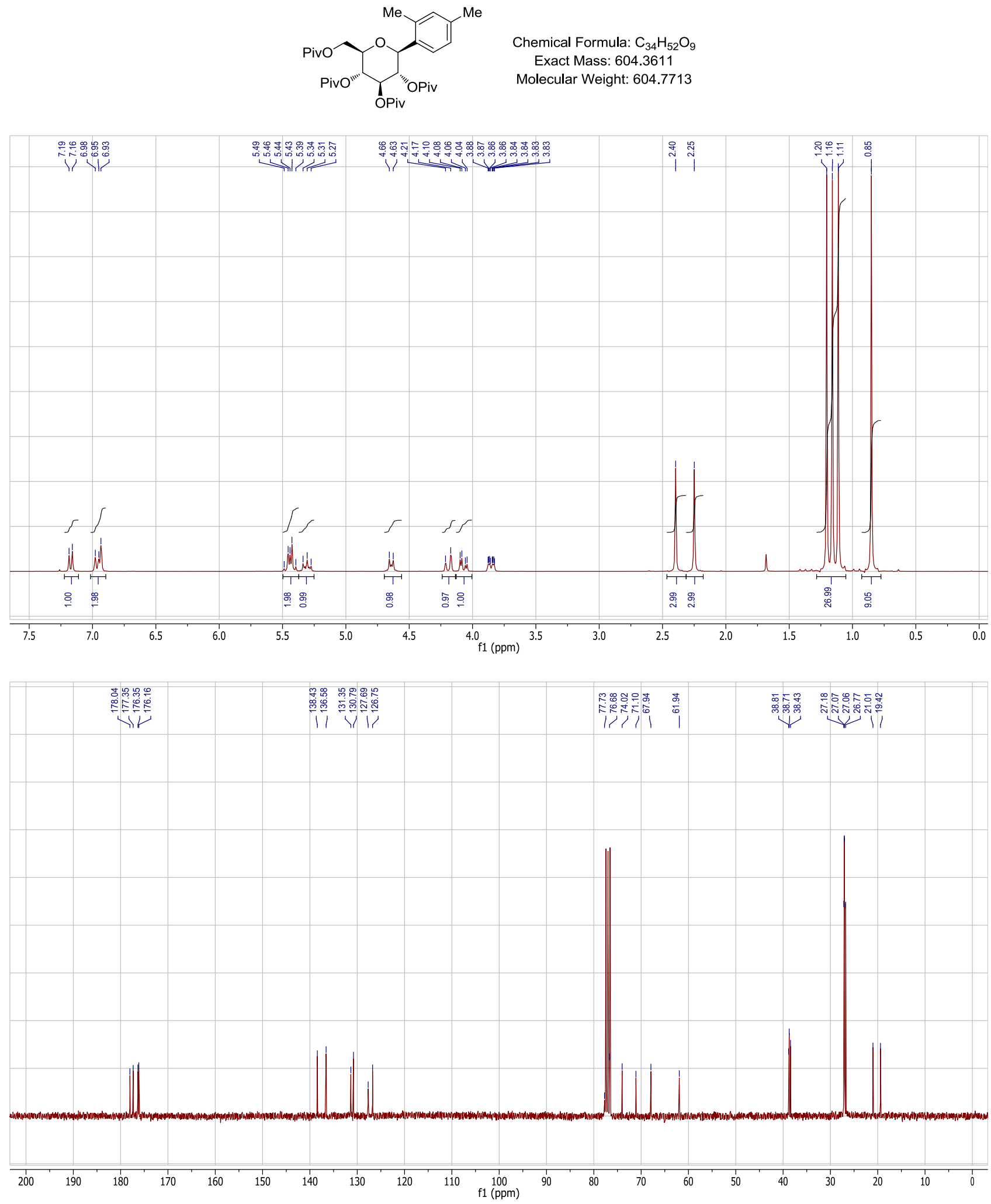
2,3,4,6-Tetra-O-pivaloyl 1-(3,5-dimethylphenyl)-1-deoxy- $\beta$-D-glucopyranose ( $\beta$-24):
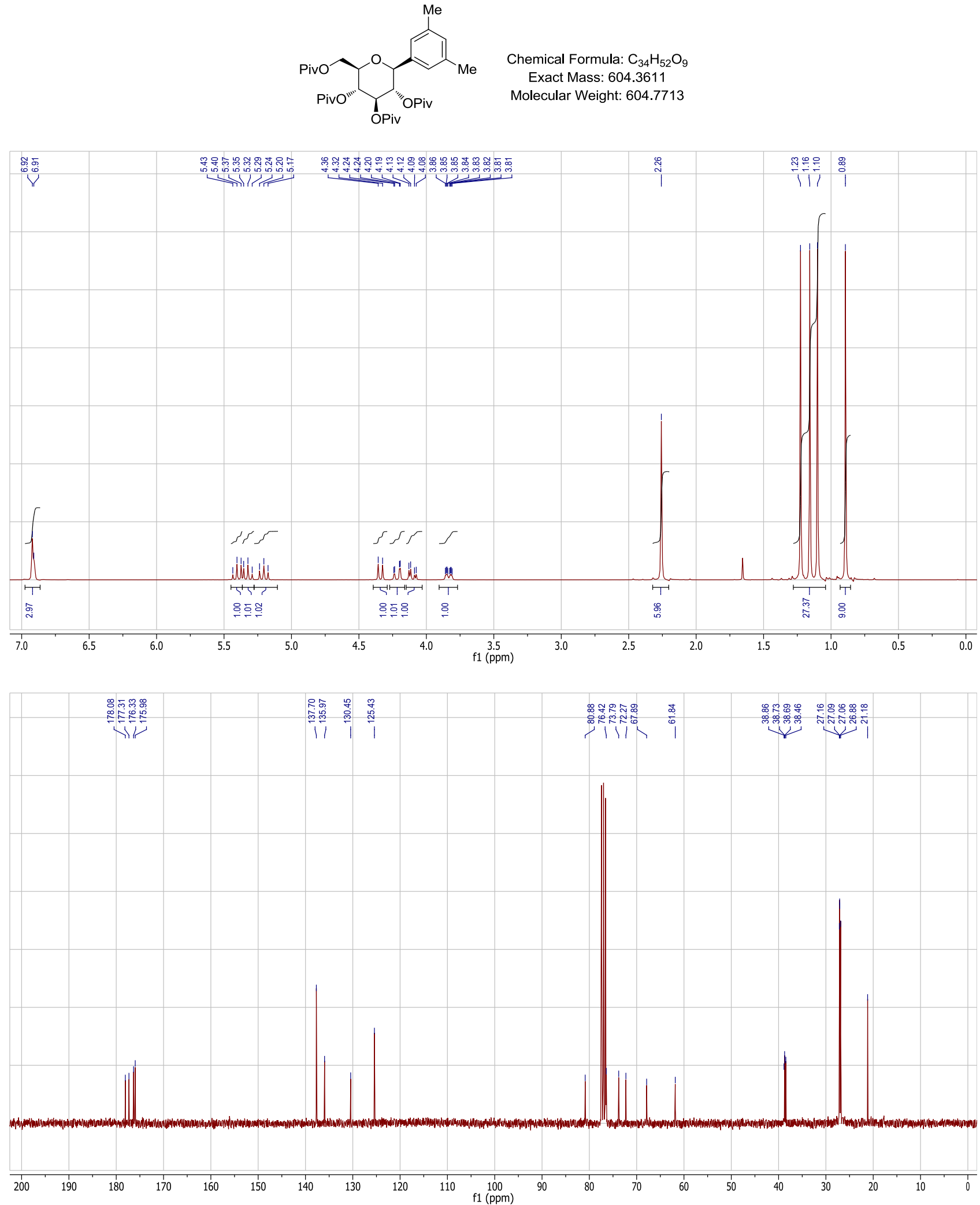
2,3,4,6-Tetra-O-pivaloyl 1-(2-thienyl)-1-deoxy- $\beta$-D-glucopyranose $(\beta-26)$ :

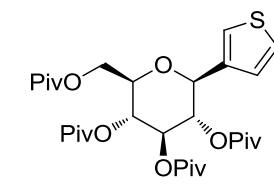

Chemical Formula: $\mathrm{C}_{30} \mathrm{H}_{46} \mathrm{O}_{9} \mathrm{~S}$

Exact Mass: 582.2863

Molecular Weight: 582.7458
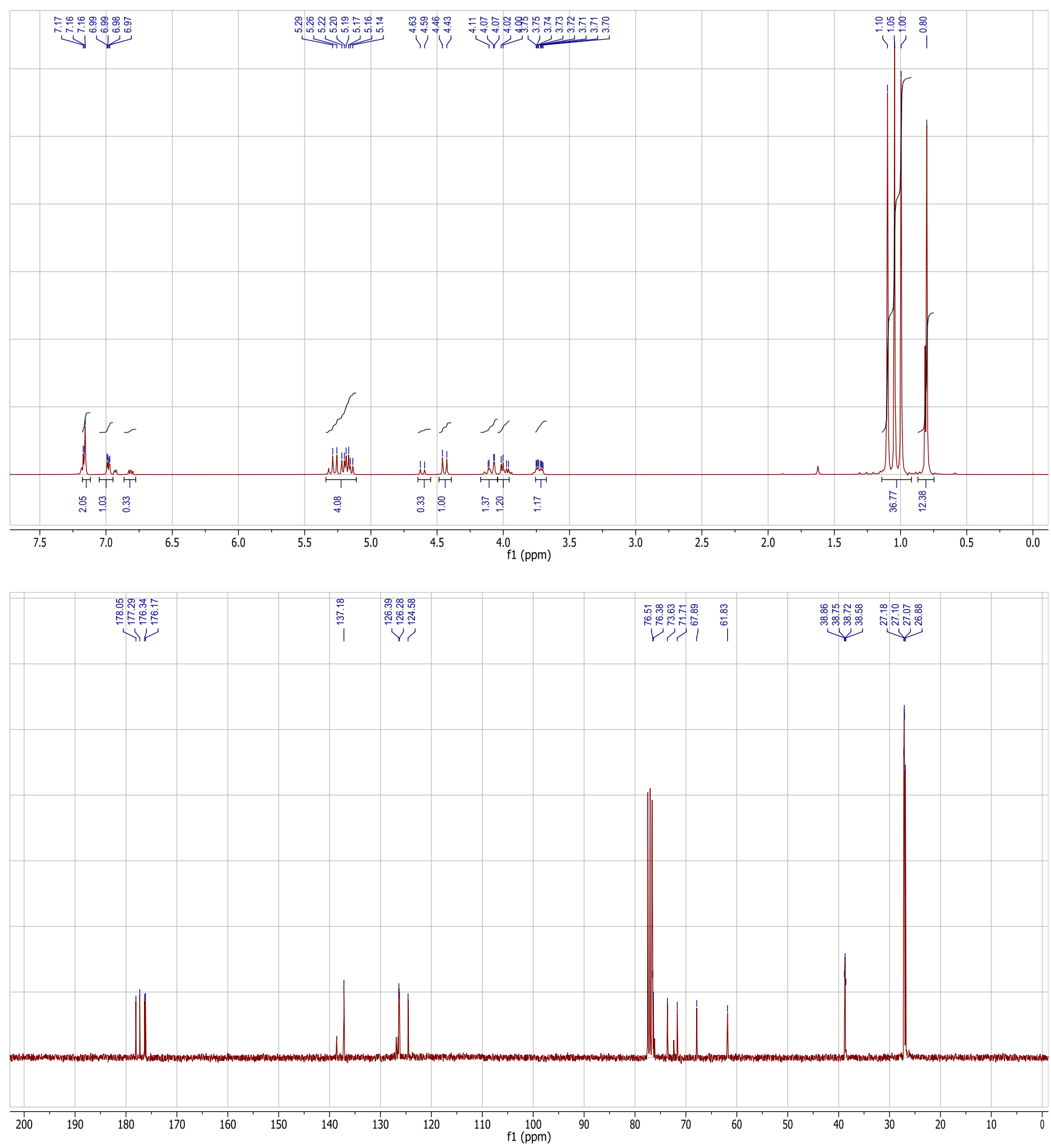


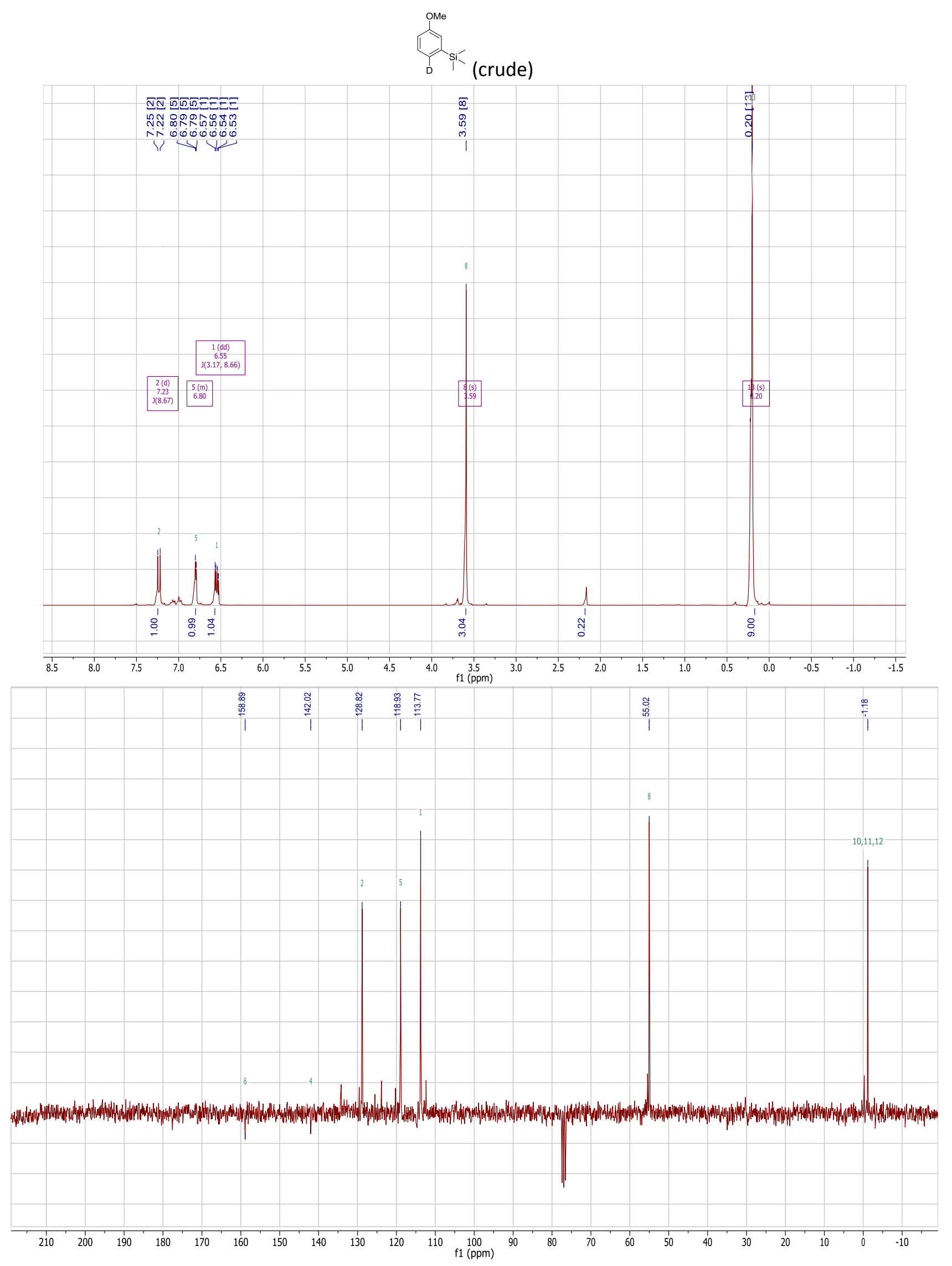


51
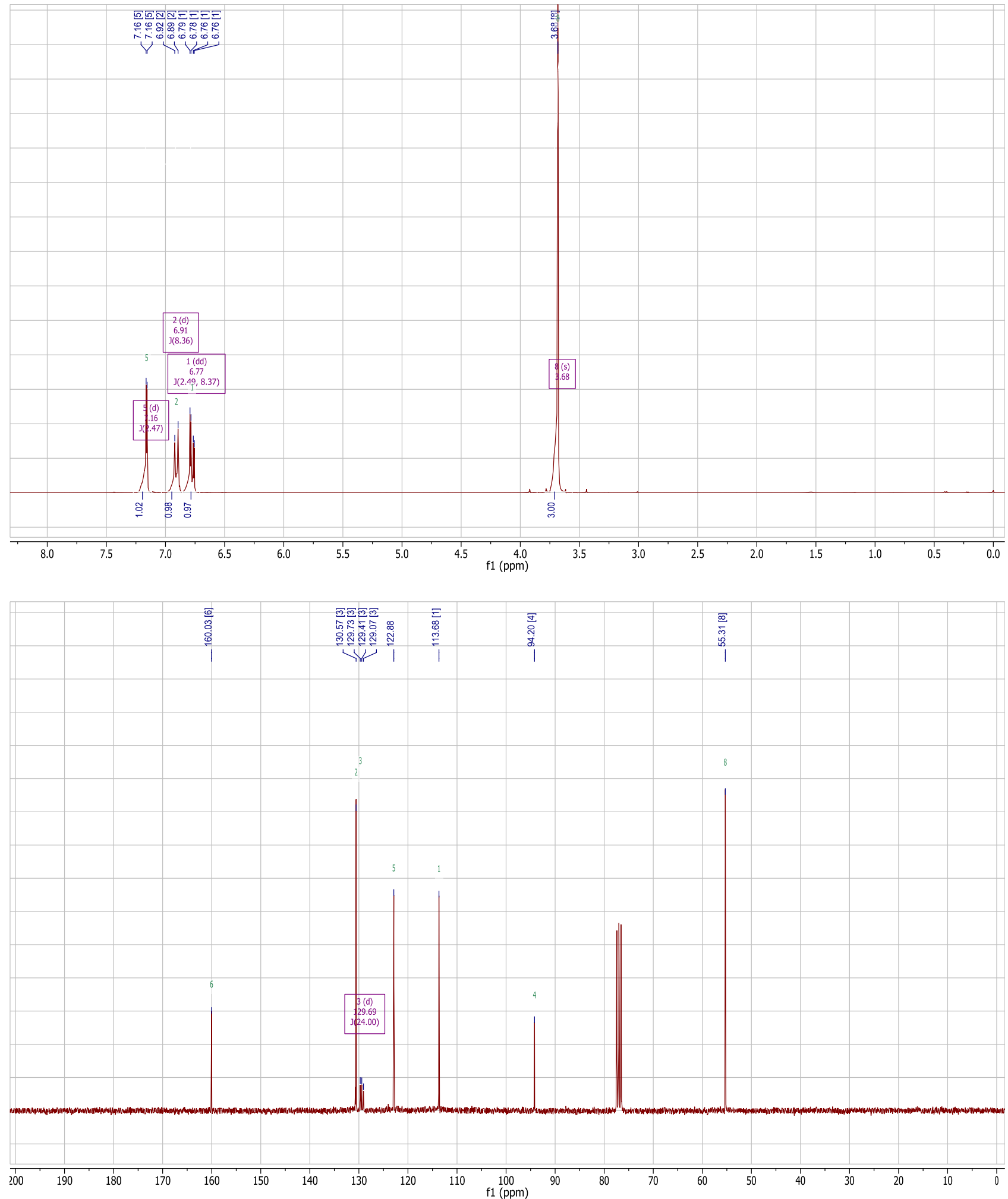
(crude)
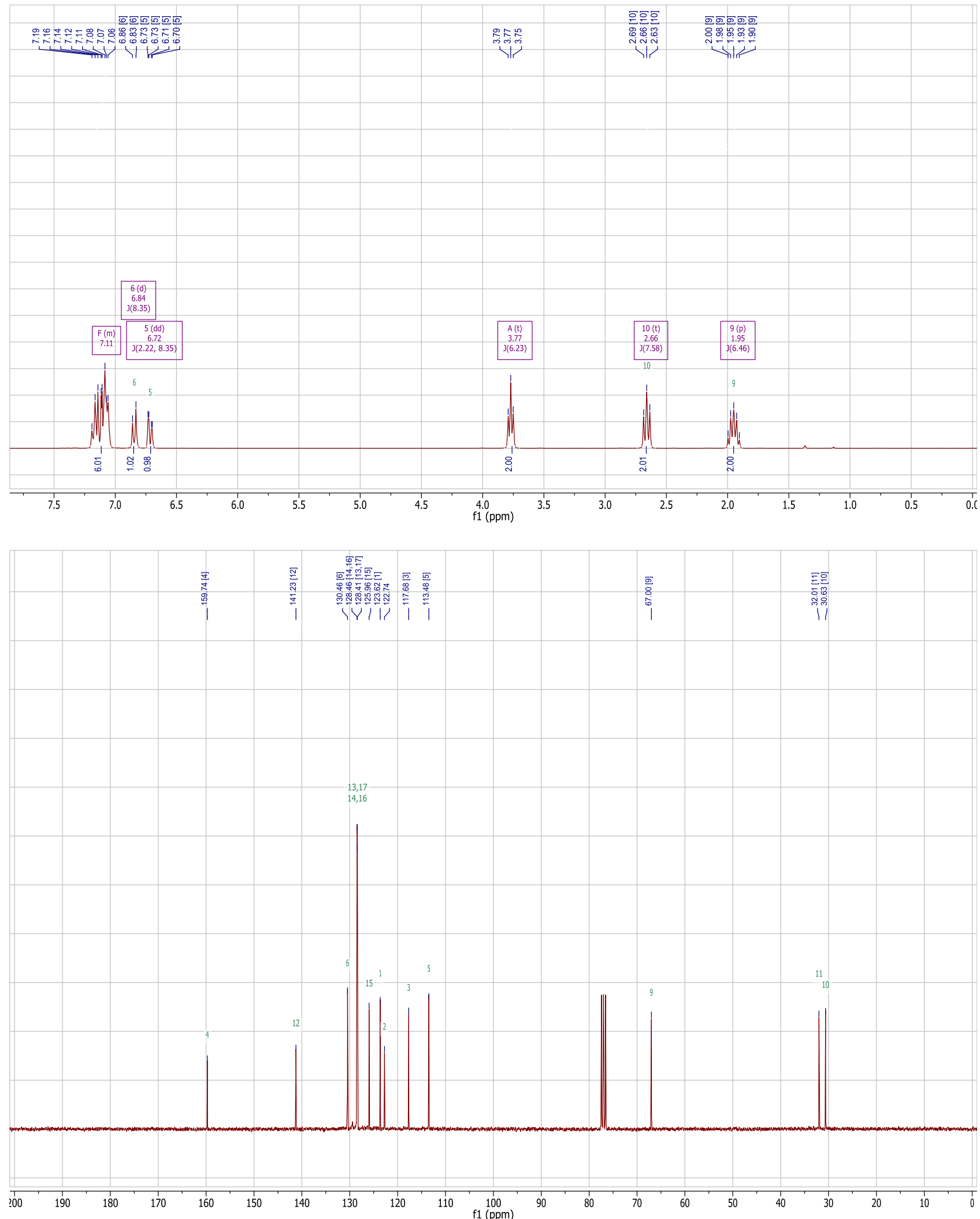
Crude

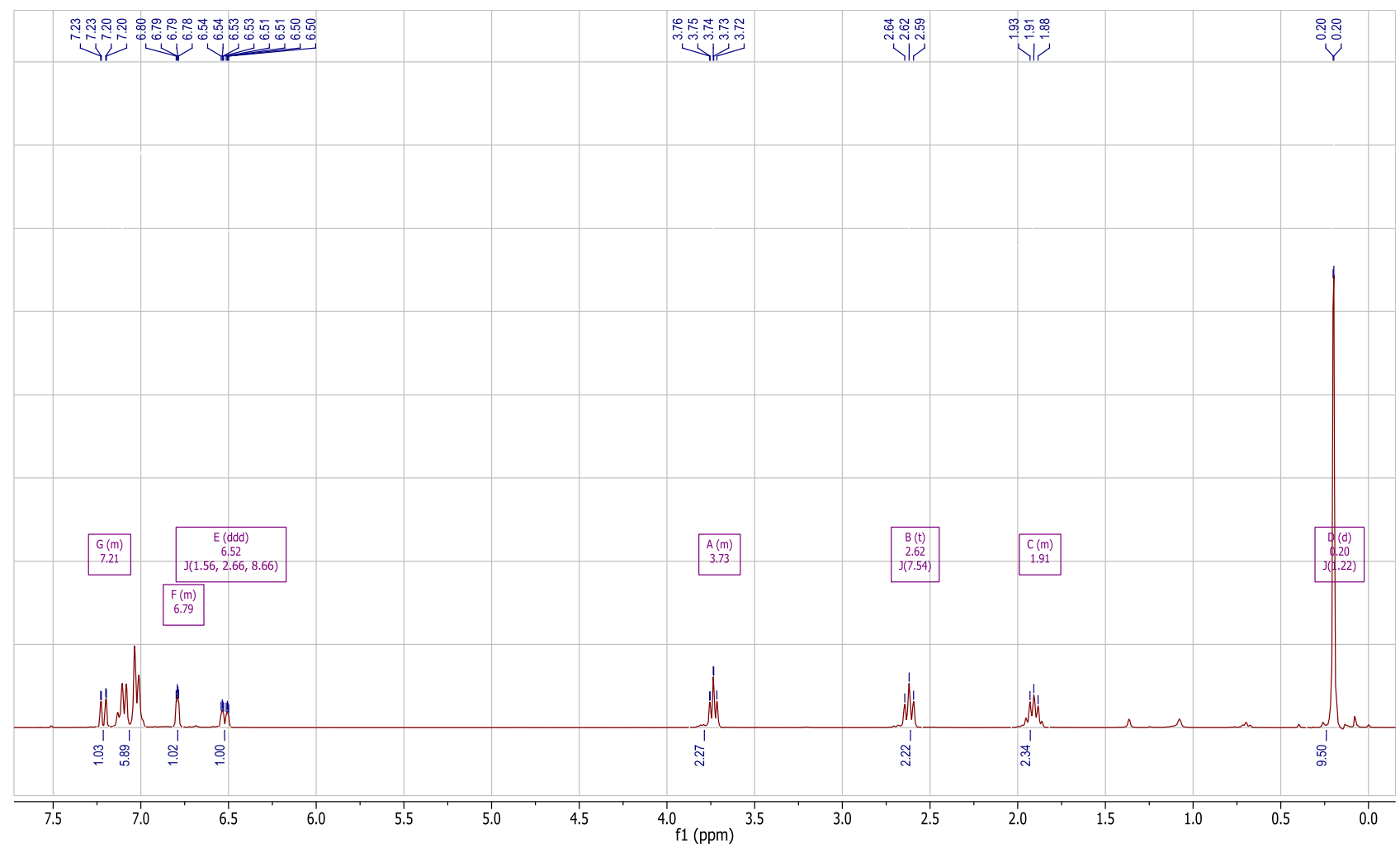

Crude

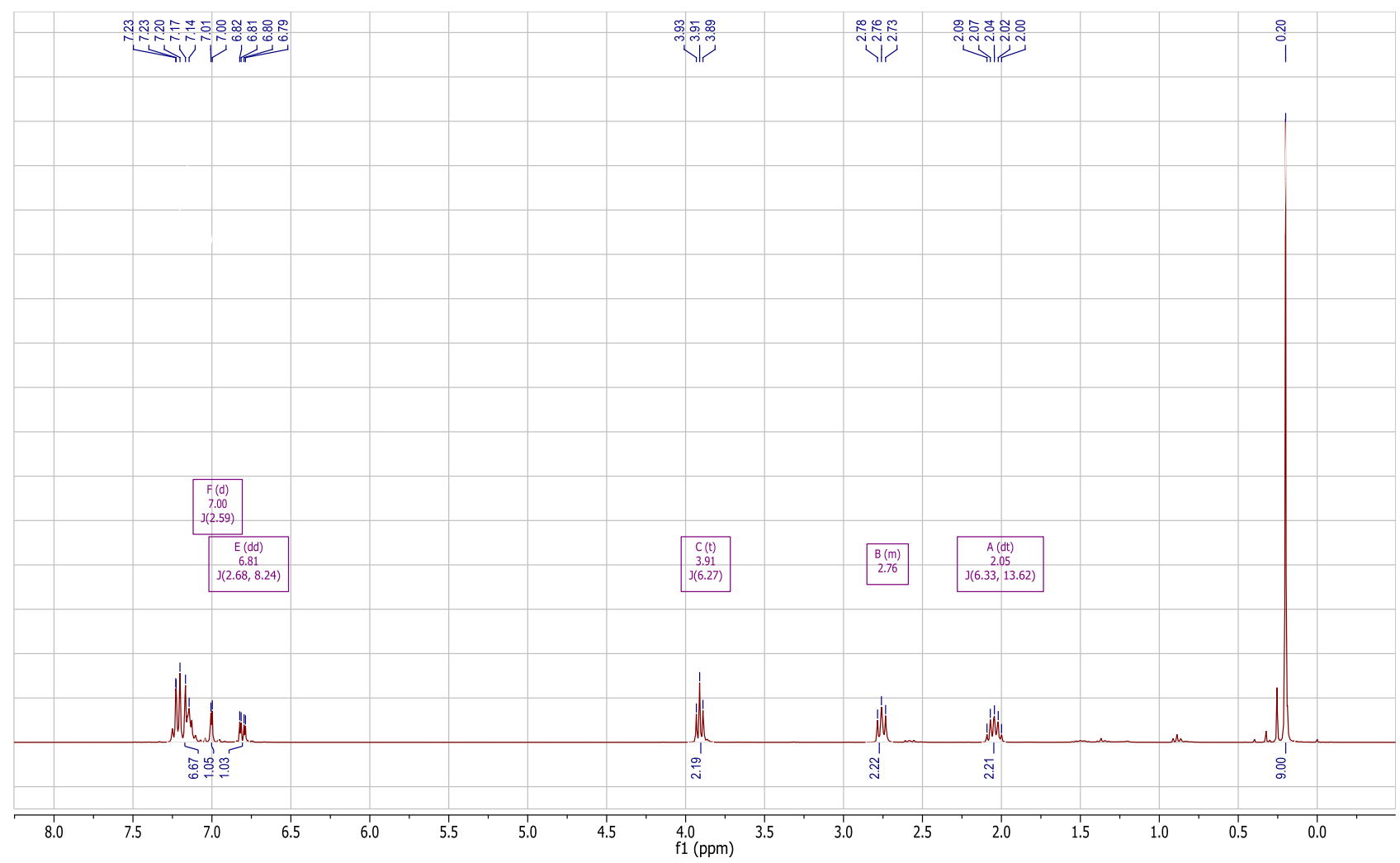


Crude

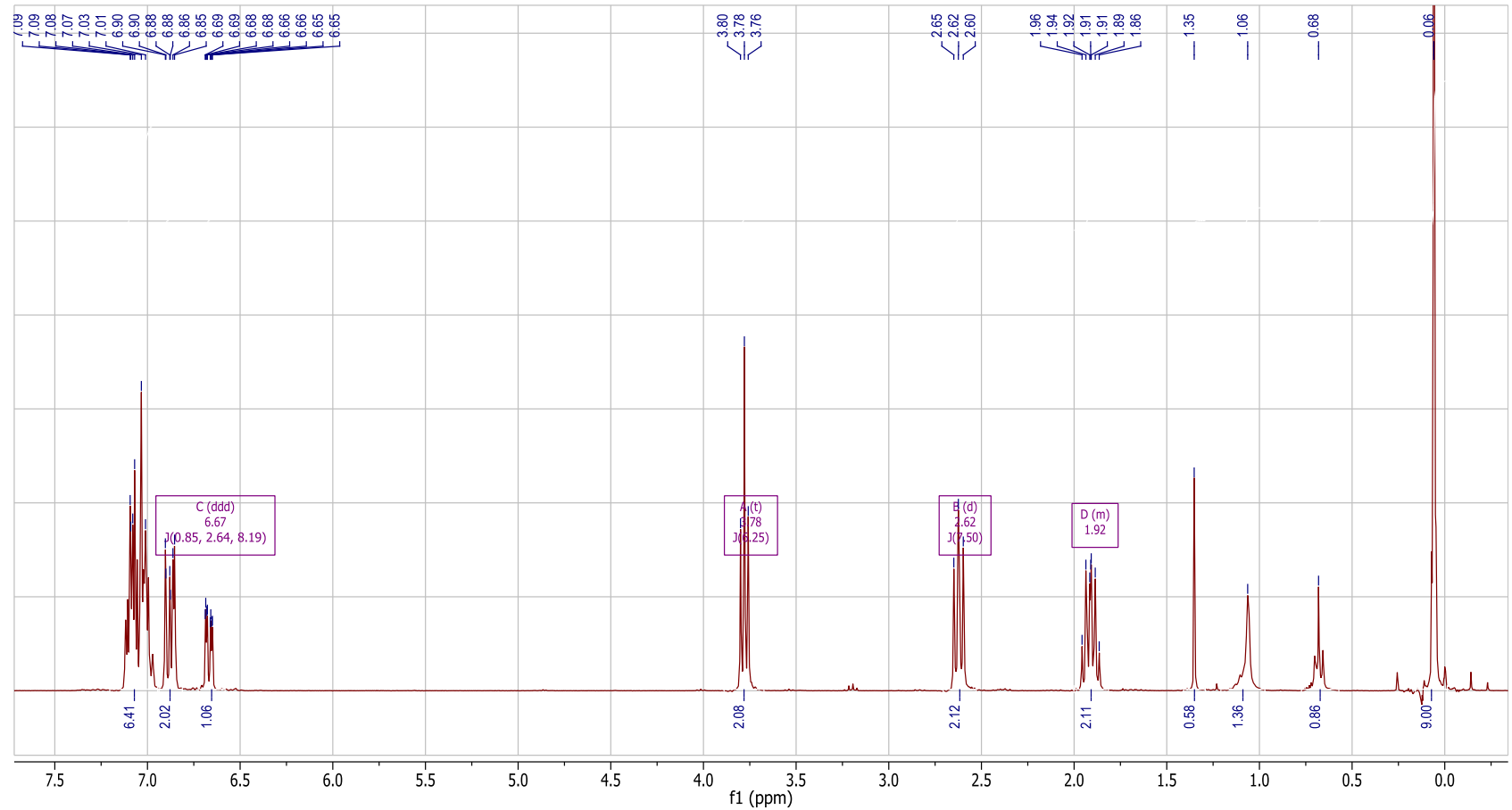


52
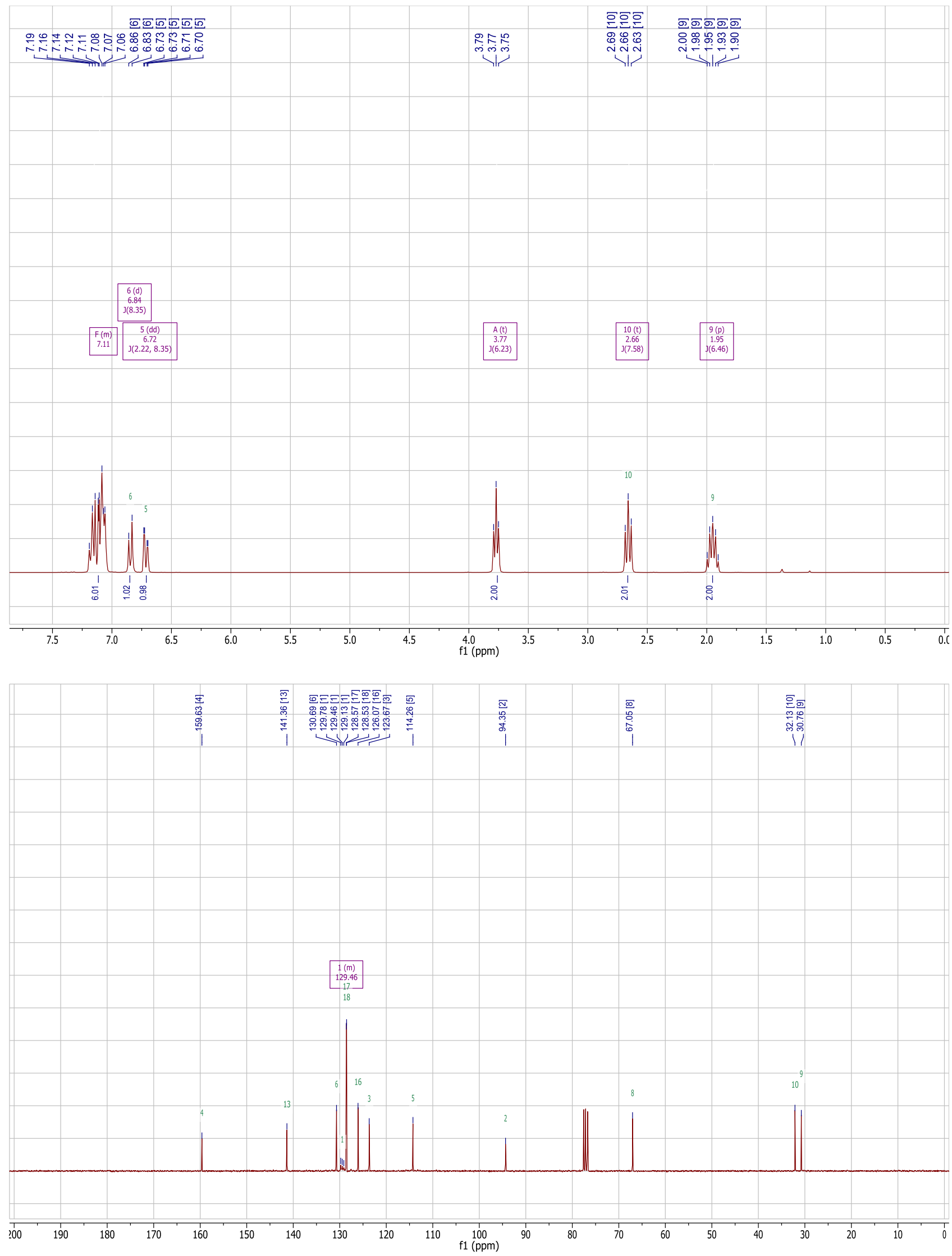

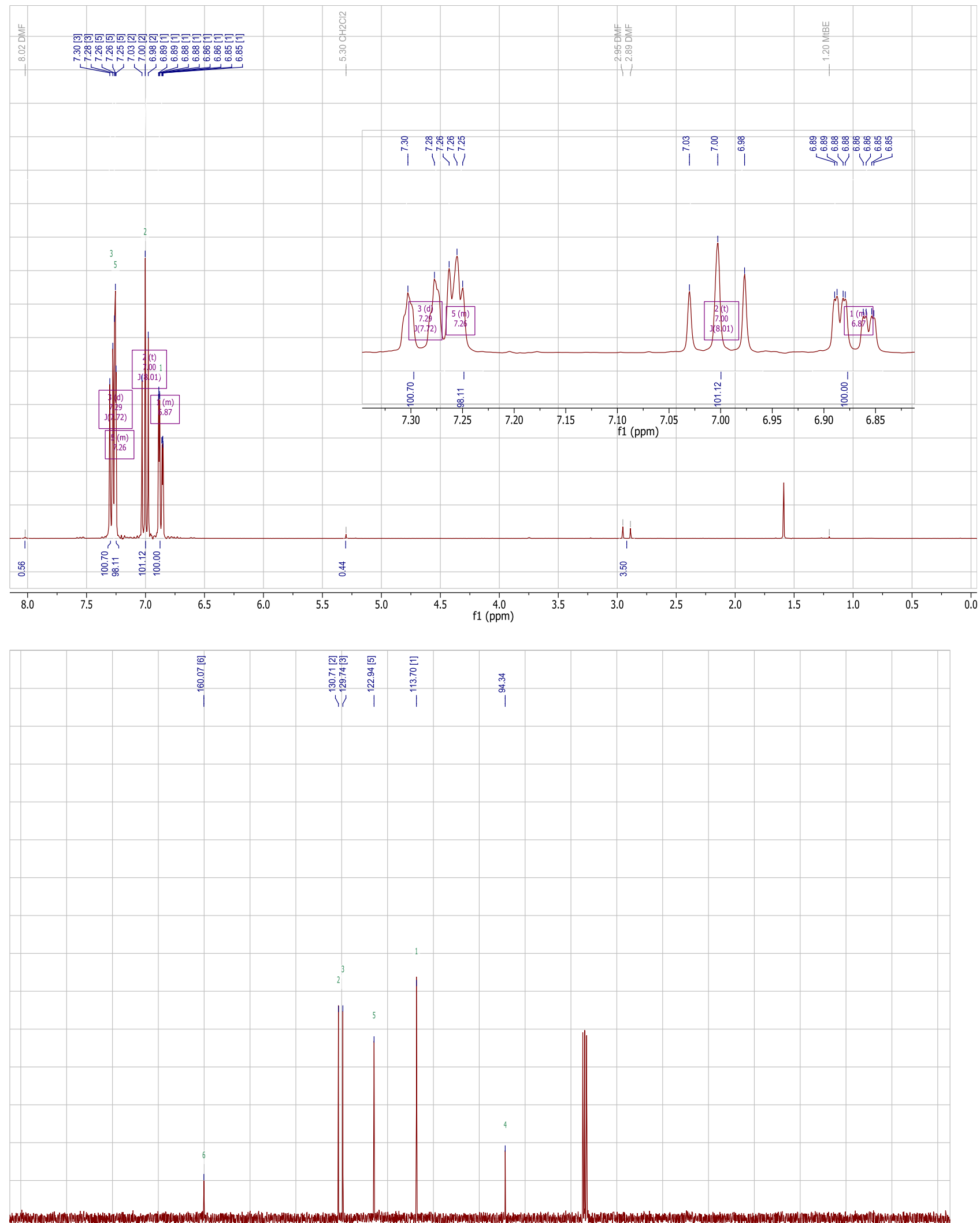
2,3,4,6-Tetra-O-pivaloyl 1-(2-deutero-5-methoxyphenyl)-1-deoxy- $\beta$-D-glucopyranose ( $\beta$-meta-32):
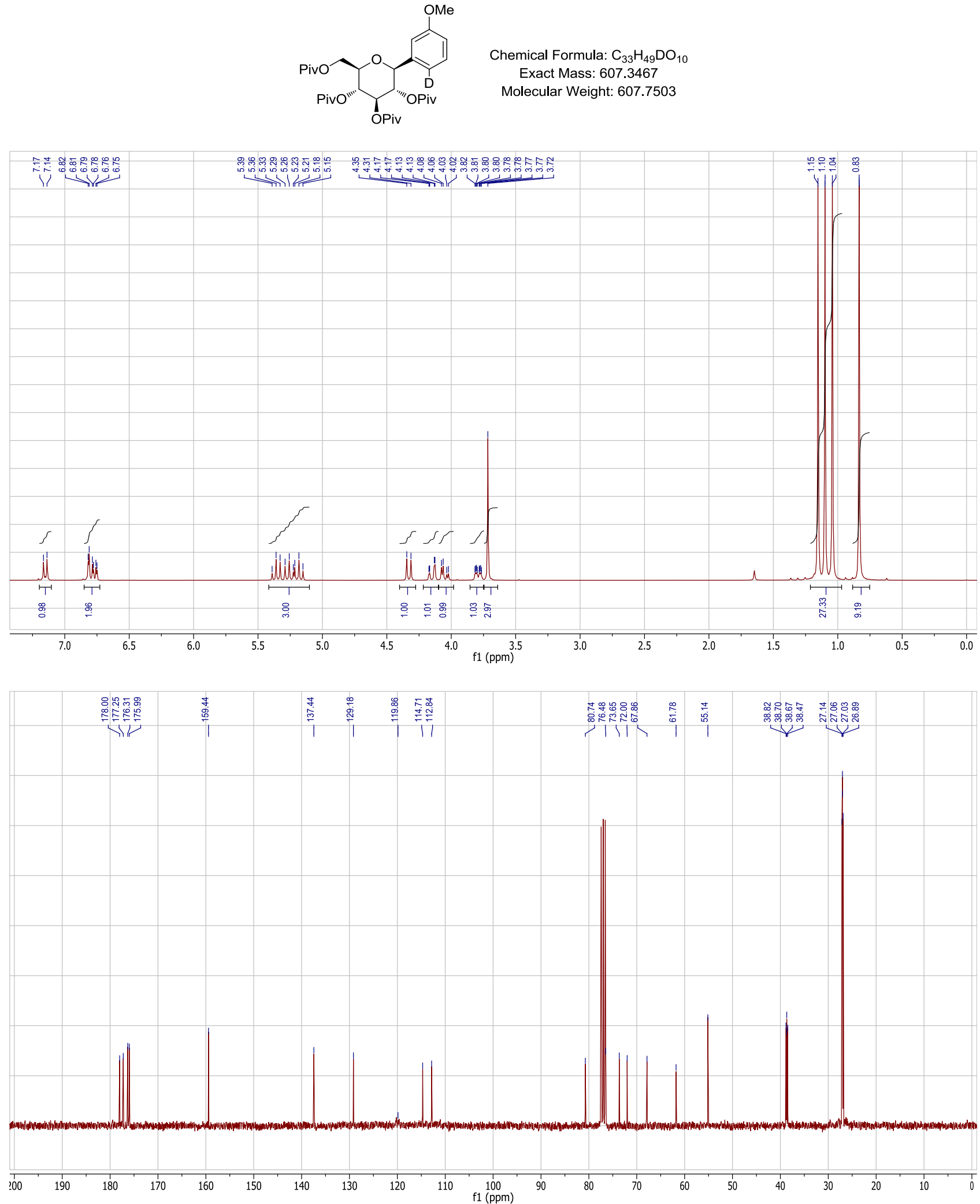
2,3,4,6-Tetra-O-pivaloyl 1-(2-deutero-4-methoxyphenyl)-1-deoxy- $\beta$-D-glucopyranose ( $\beta$-para-32)

(Reaction quenched with MeOD)
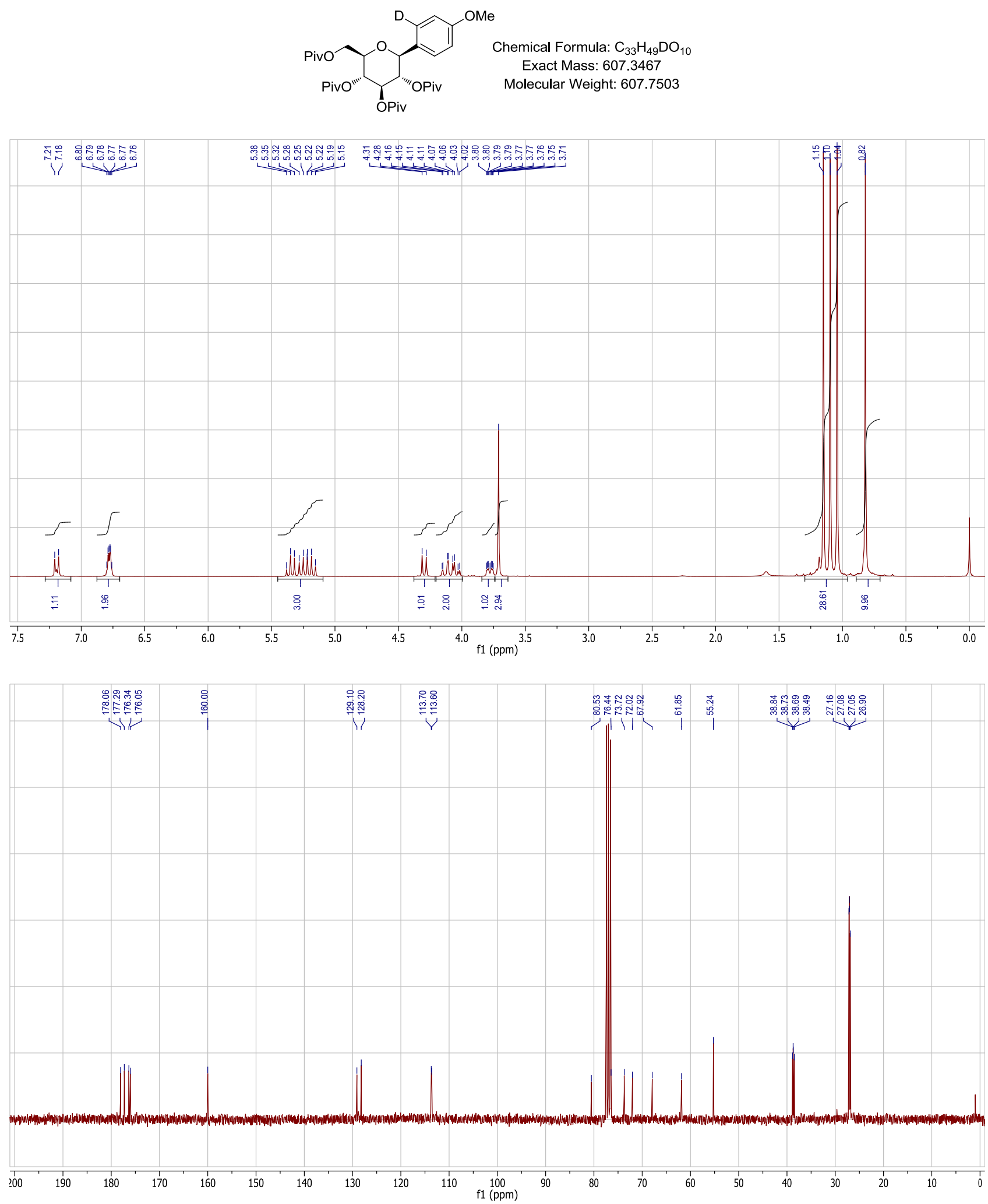
2,3,4,6-Tetra-O-pivaloyl 1-(2-deutero-4-methoxyphenyl)-1-deoxy- $\beta$-D-glucopyranose $\quad(\beta$-para-32) + 2,3,4,6-Tetra-O-pivaloyl 1-(4-anisyl)-1-deoxy- $\beta$-D-glucopyranose ( $\beta$-para-6)

(Reaction quenched with $\mathrm{NH}_{4} \mathrm{Cl}$ )
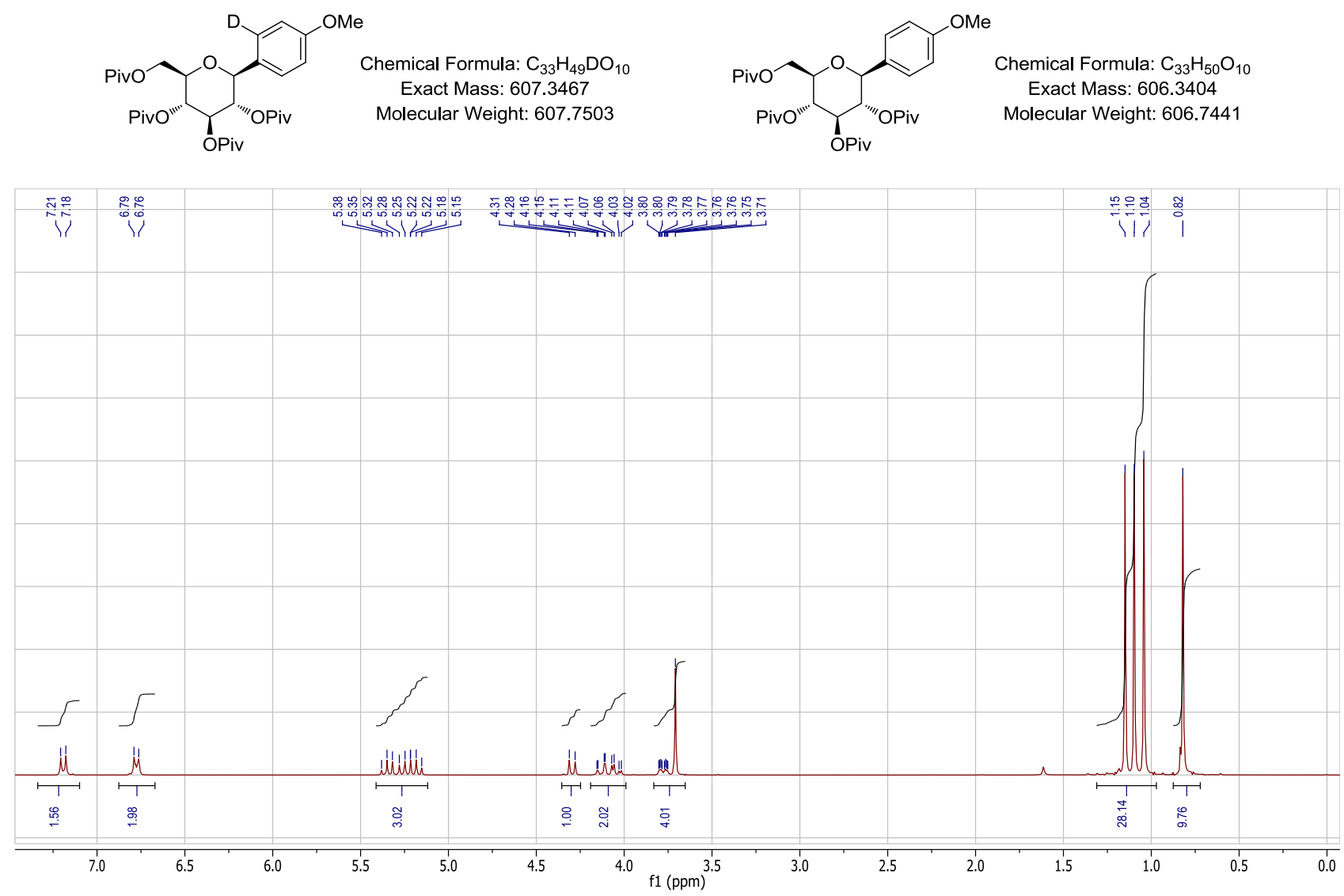
2,3,4,6-Tetra-O-pivaloyl 1-(2-iodo-4-methoxyphenyl)-1-deoxy- $\beta$-D-glucopyranose ( $\beta$-para-33)
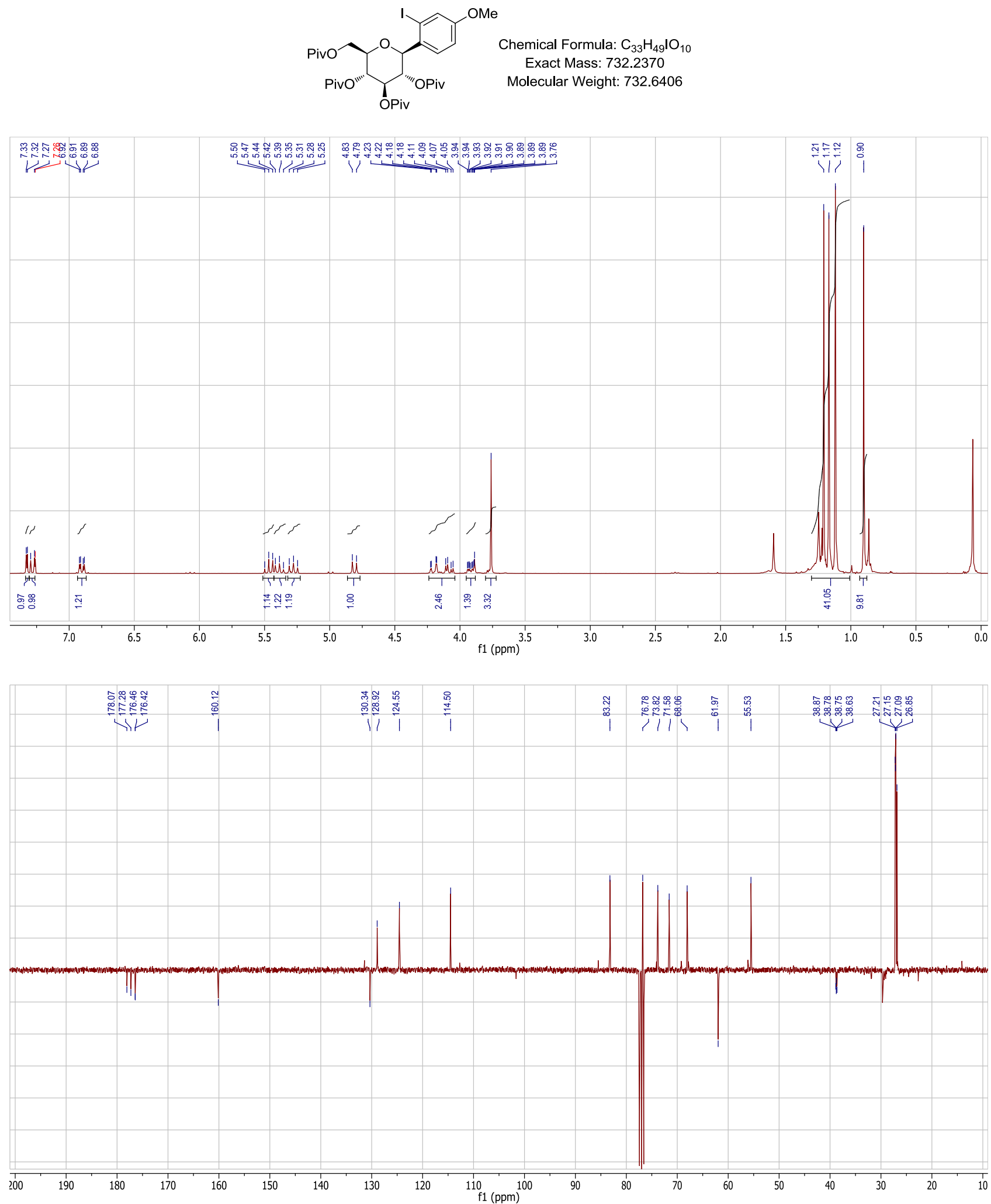
2,3,4,6-Tetra-O-pivaloyl 1-(4-(3-phenylpropoxy)phenyl)-1-deoxy- $\beta$-D-glucopyranose ( $\beta$-para-38):

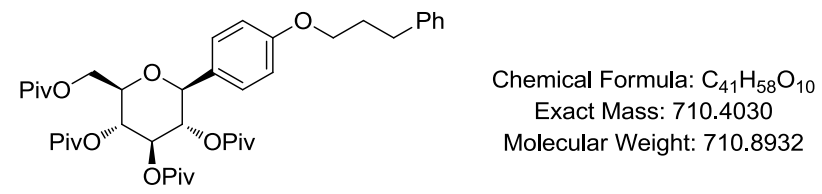

(sample synthesized using non-deuterated arylzinc reagent)
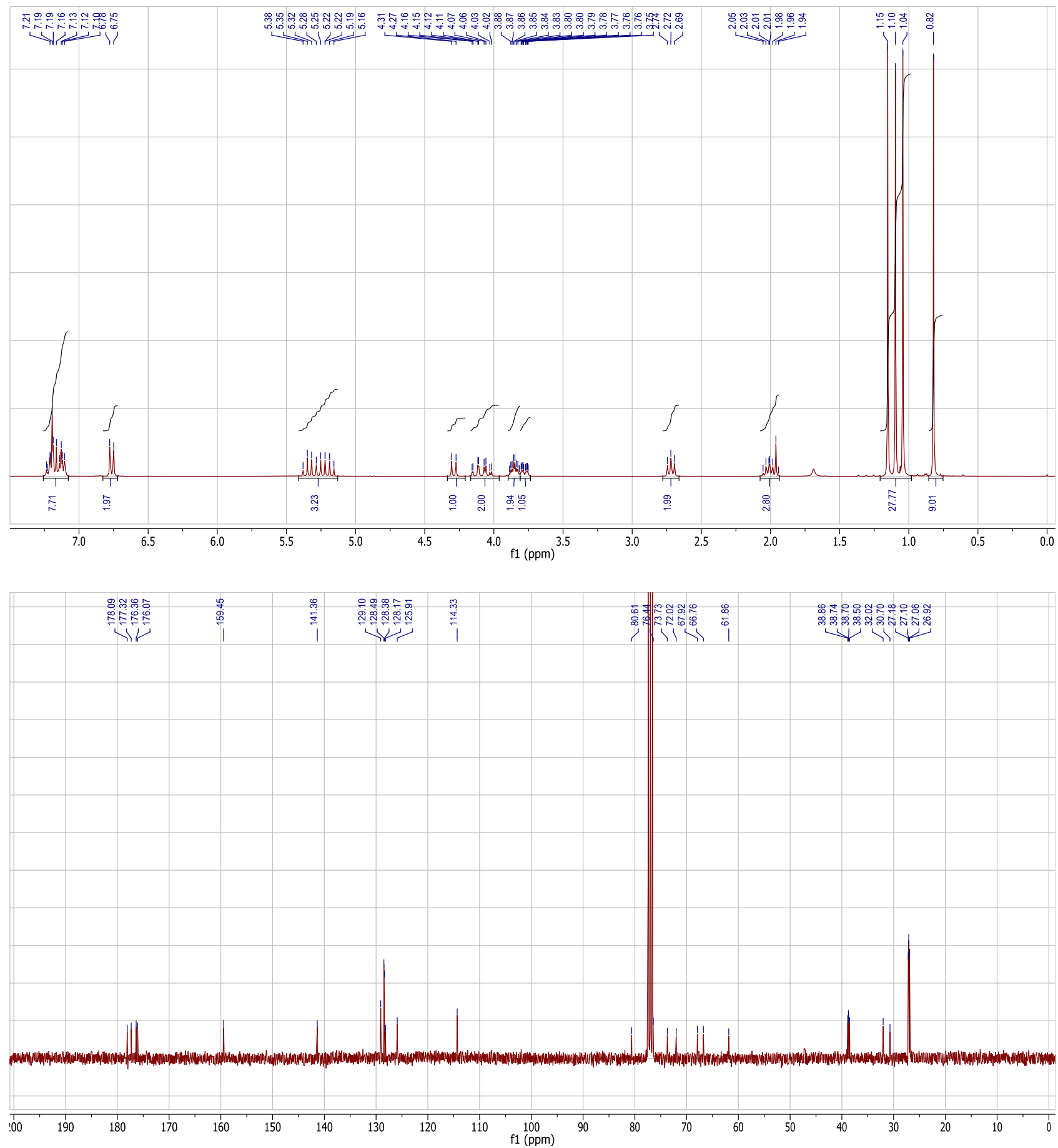
4,4'-((4-methoxyphenyl)methylene)bis(methylbenzene) (42)

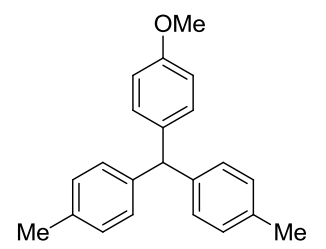

Chemical Formula: $\mathrm{C}_{22} \mathrm{H}_{22} \mathrm{O}$

Exact Mass: 302.1671

Molecular Weight: 302.4095
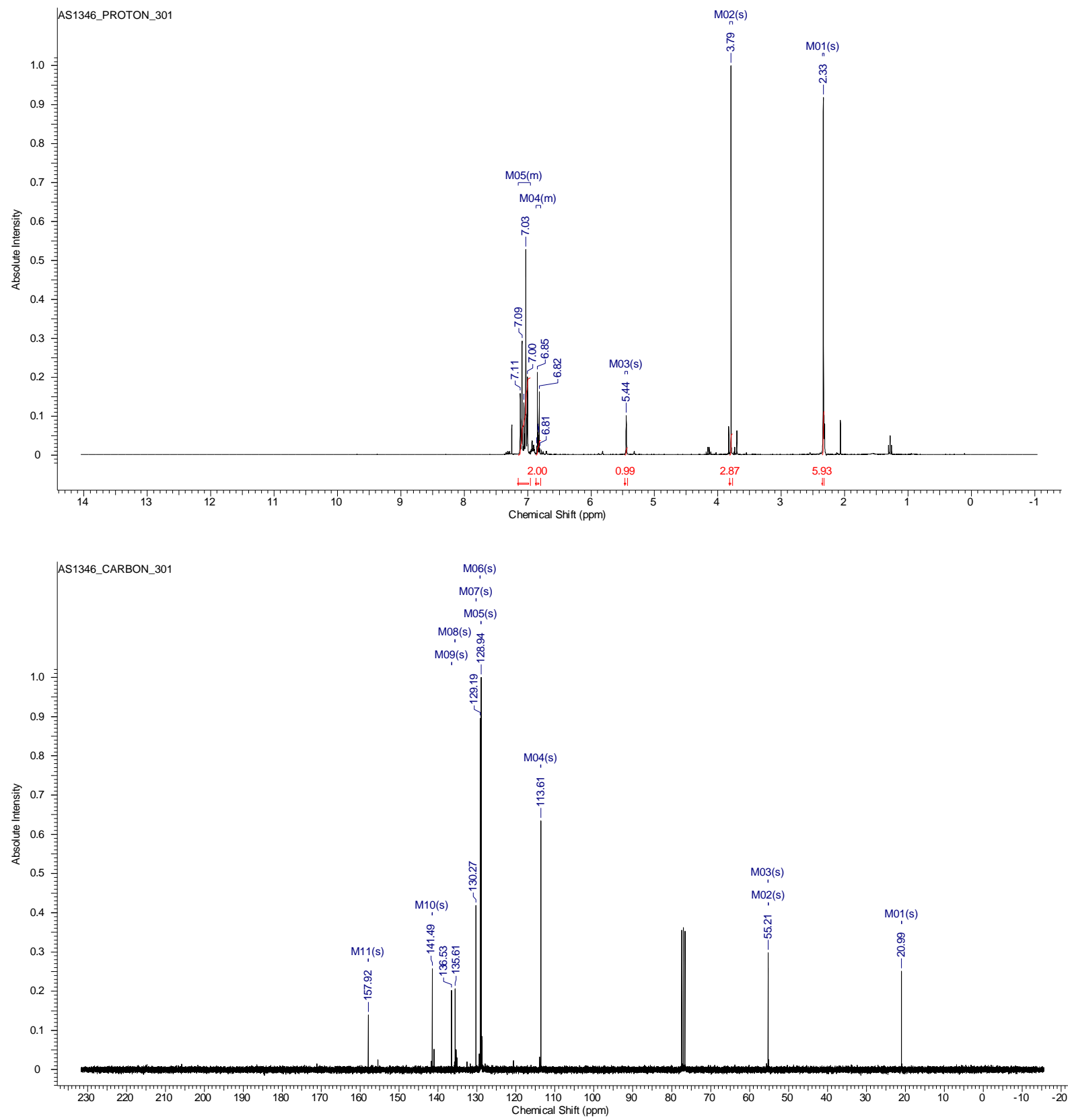
Ethyl 2'-(di-p-tolylmethyl)-5'-methoxy-[1,1'-biphenyl]-4-carboxylate (45)

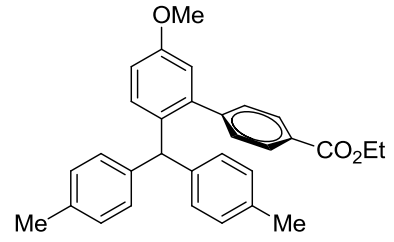

Chemical Formula: $\mathrm{C}_{31} \mathrm{H}_{30} \mathrm{O}_{3}$

Exact Mass: 450.2195

Molecular Weight: 450.5681
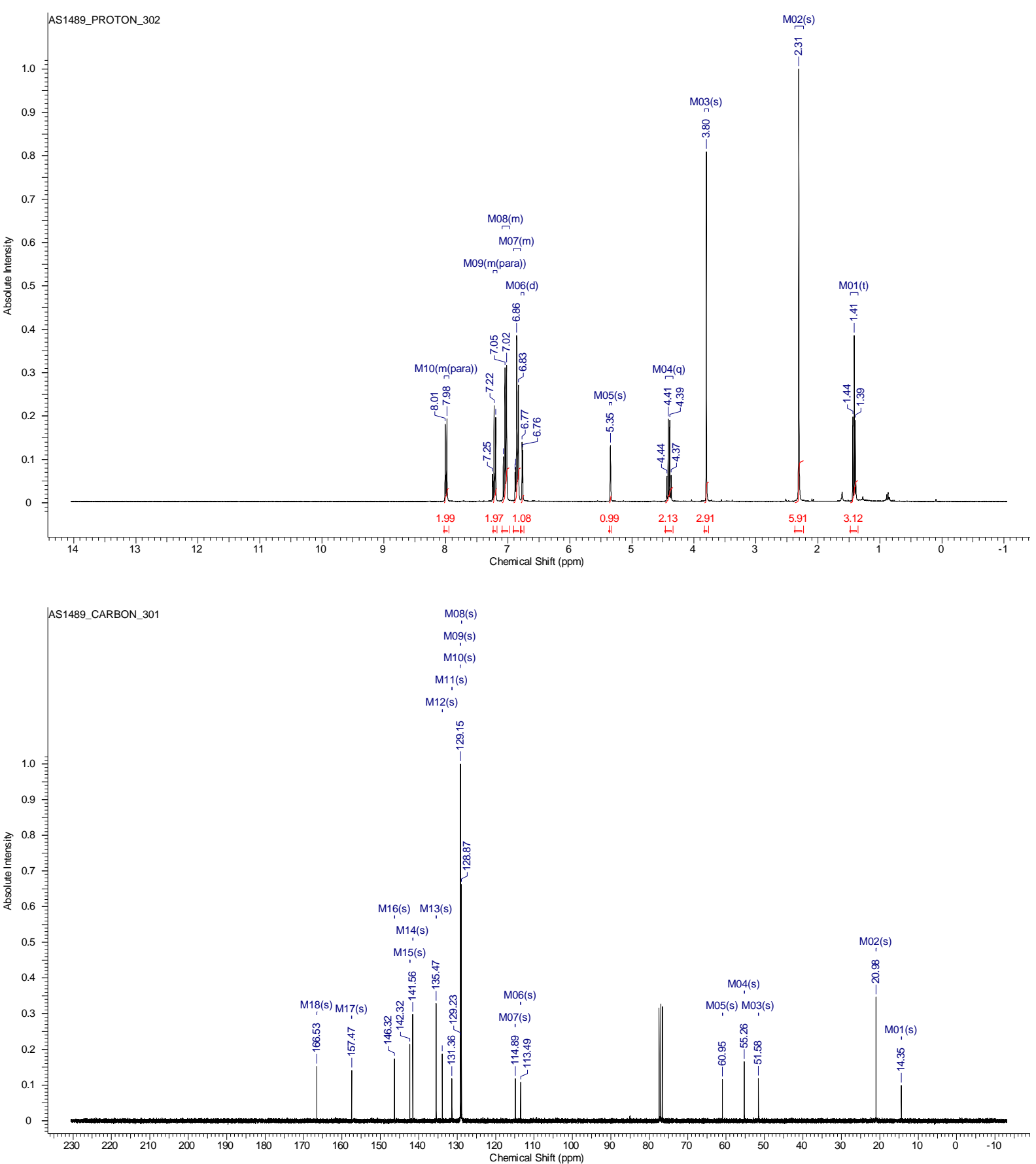


\section{(2-(Di-p-tolylmethyl)-5-methoxyphenyl)(phenyl)methanol (46)}

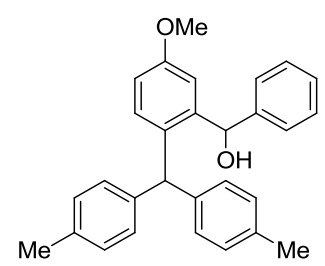

Chemical Formula: $\mathrm{C}_{29} \mathrm{H}_{28} \mathrm{O}_{2}$

Exact Mass: 408.2089

Molecular Weight: 408.5314
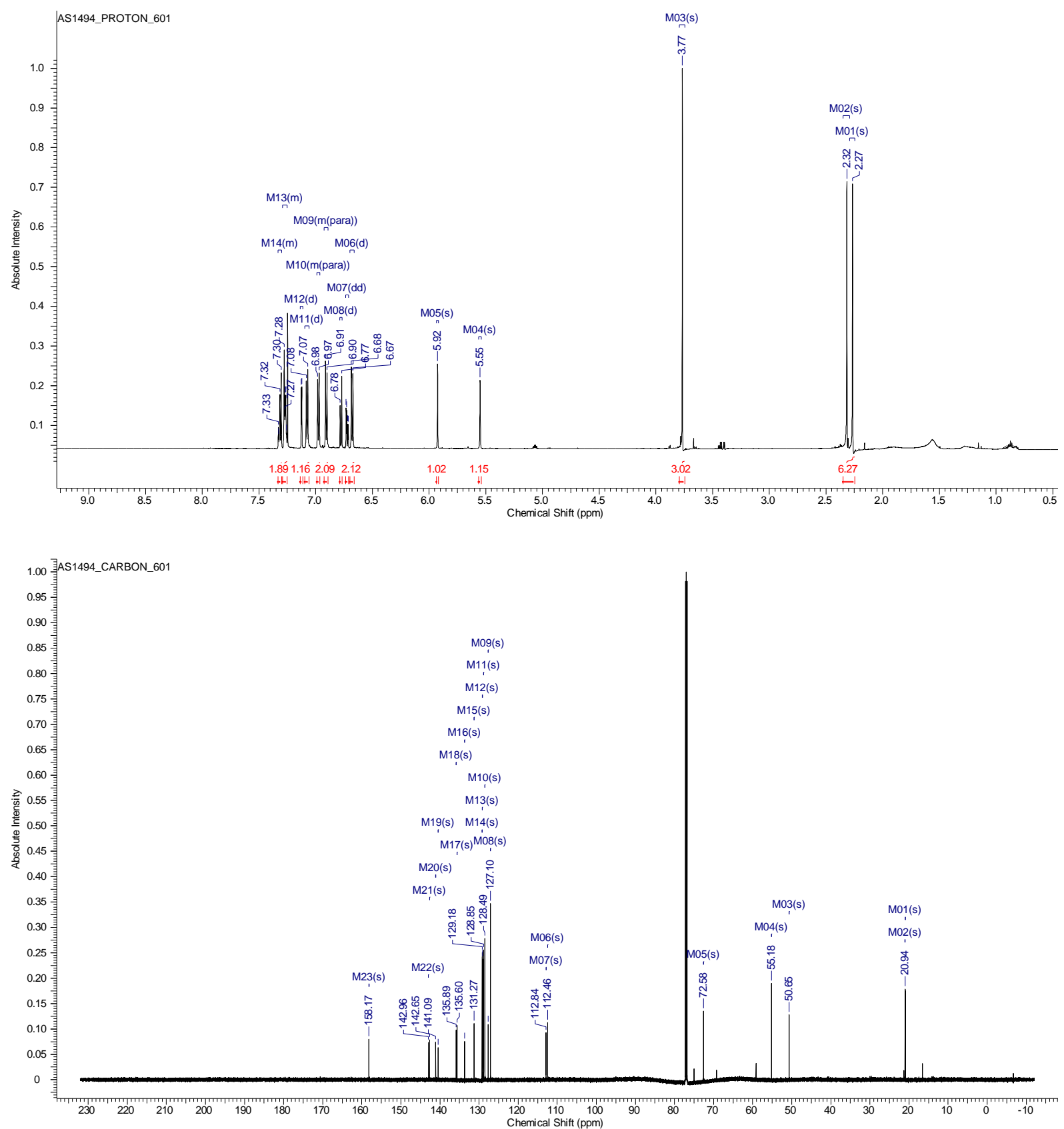
(2-(Di-p-tolylmethyl)-5-methoxyphenyl)(thiophen-2-yl)methanone (47)<smiles>COc1ccc(C(c2ccc(C)cc2)c2ccc(OC)cc2C(=O)c2cccs2)cc1</smiles>

Chemical Formula: $\mathrm{C}_{27} \mathrm{H}_{24} \mathrm{O}_{2} \mathrm{~S}$ Exact Mass: 412.1497

Molecular Weight: 412.5433
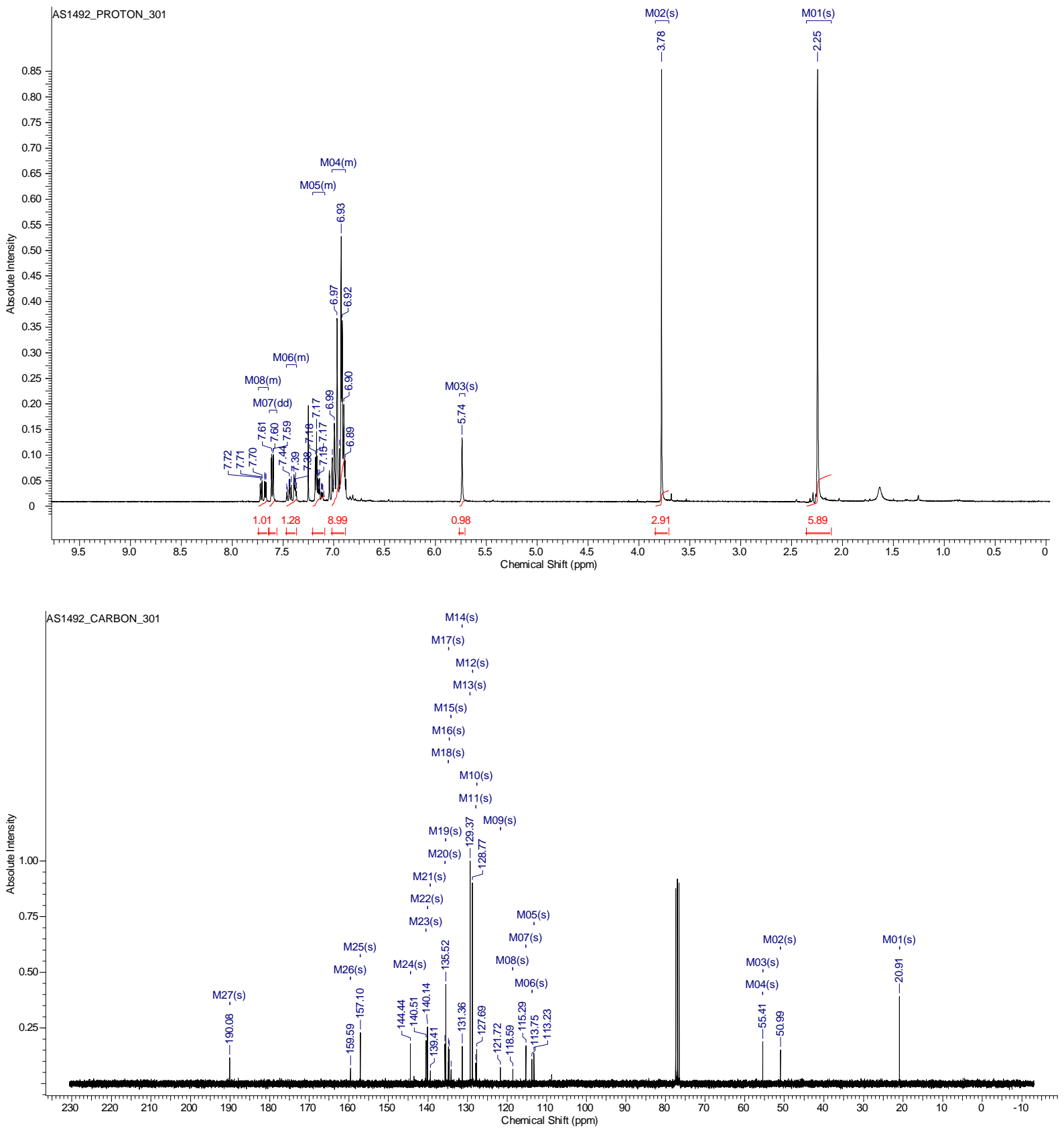
Ethyl 2-(2-(di-p-tolylmethyl)-5-methoxybenzyl)acrylate (48)<smiles>C=C(Cc1cc(OC)ccc1C(c1ccc(C)cc1)c1ccc([N+](=O)[O-])cc1)C(=O)OC</smiles>

Chemical Formula: $\mathrm{C}_{28} \mathrm{H}_{30} \mathrm{O}_{3}$ Exact Mass: 414.2195

Molecular Weight: 414.5360
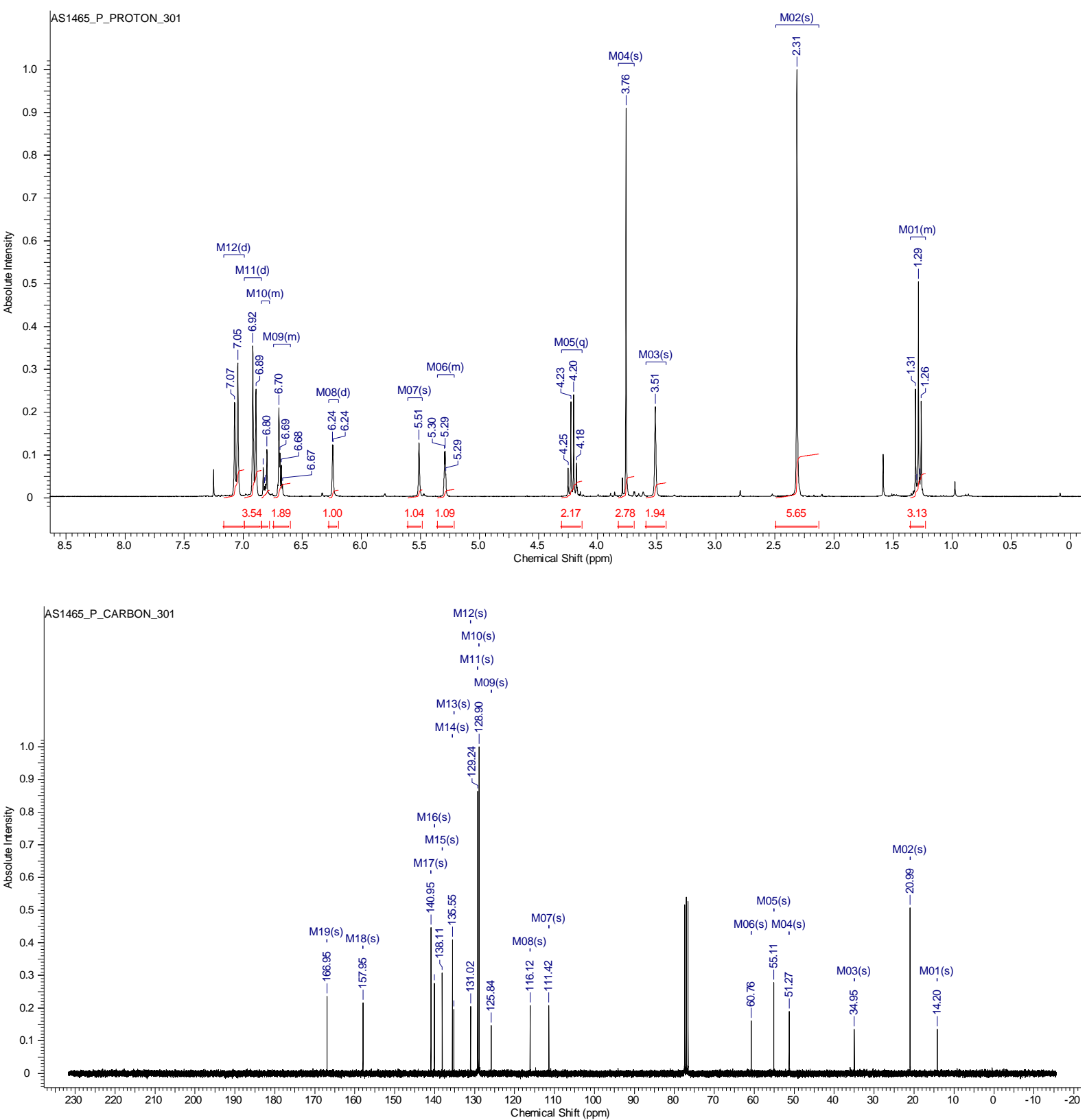